\title{
OMITTING TYPES IN FRAGMENTS AND EXTENSIONS OF FIRST ORDER LOGIC
}

\begin{abstract}
Fix $2<n<\omega$. Let $L_{n}$ denote first order logic restricted to the first $n$ variables. Using the machinery of algebraic logic, positive and negative results on omitting types are obtained for $L_{n}$ and for infinitary variants and extensions of $L_{\omega, \omega}$.

Keywords: Algebraic logic, multimodal logic, omitting types, completions.

\section{Introduction}

Let $\mathfrak{L}$ be an extension or reduct or variant of first order logic, like first logic itself possibly without equality, $L_{n}$ as defined in the abstract with $2<n<\omega, L_{\omega_{1}, \omega}, L_{\omega}$ as defined in $[10, \S 4.3], \ldots$, etc. An omitting types theorem for $\mathfrak{L}$, briefly an OTT, is typically of the form 'A countable family of non-isolated types in a countable $\mathfrak{L}$ theory $T$ can be omitted in a countable model of $T$. From this it directly follows, that if a type is realizable in every model of a countable theory $T$, then there should be a formula consistent with $T$ that isolates this type. A type is simply a set of formulas $\Gamma$ say. The type $\Gamma$ is realizable in a model if there is an assignment that satisfies (uniformly) all formulas in $\Gamma$. Finally, $\phi$ isolates $\Gamma$ means that $T \vdash \phi \rightarrow \psi$ for all $\psi \in \Gamma$. What Orey and Henkin proved is that the OTT holds for $L_{\omega, \omega}$ when such types are finitary meaning that they all consist of $n$-variable formulas for some $n<\omega$. For $L_{n}$, as defined in the abstract, the situation turns out drastically different. It is known [2] that the OTT fails in the following (strong) sense. For every $2<n \leq l<\omega$, there is a
\end{abstract}

Presented by: Janusz Ciuciura

Received: May 17, 2019

Published online: May 28, 2021

(c) Copyright by Author(s), Łódź 2021

(C) Copyright for this edition by Uniwersytet Łódzki, Łódź 2021 
countable and complete $L_{n}$ theory $T$, and a type that is realizable in every model of $T$, but cannot be isolated by a formula using $l$ variables.

In this paper we prove other negative OTTs for $L_{n}$ when types are required to be omitted with respect to certain generalized semantics.

By imposing extra conditions on theories and / or types required to be omitted (like quantifier elimination and maximality, respectively), we obtain positive OTTs for $L_{n}$ theories; addressing possibly uncountably many types. Also, we study OTTs for algebraizable extensions of $L_{\omega, \omega}$, namely, the (algebraizable) so-called infinitary logics of infinitary relations studied extensively in $[10, \S 4.3]$. In this context, we prove negative results on OTTs. Here semantics are the usual Tarskian semantics respecting commutativity of cylindrifiers. Sometimes such logics are referred to as typless logics; the adjective typless pointing out to dropping the arity of relation symbols in their formalism.

Conversely, we prove positive OTTs for logics corresponding to variants of $\omega$-dimensional polyadic algebras with equality $\left(\mathrm{PEA}_{\omega} \mathrm{s}\right)$ with equality studied in $[8,18]$ by taking reducts and/or weakening the axioms of PEA . $_{\text {. }}$. In the logics studied in [8], Tarskian semantics are relativized, and consequently we do not have full fledged commutativity of cylindrifiers. The logic studied in [18] can be regarded as a classical algebraizable extension of $L_{\omega, \omega}$ without equality; here by classical we understand that Tarskian semantics are preserved in such extensions.

We follow the notation of [1] which is in conformity with the notation in the monograph [10]. In particular, for any pair of ordinal $\alpha<\beta, \mathrm{CA}_{\alpha}$ stands for the class of cylindric algebras of dimension $\alpha, \mathrm{RCA}_{\alpha}$ denotes the class of representable $\mathrm{CA}_{\alpha} \mathrm{s}$ and $\mathrm{Nr}_{\alpha} \mathrm{CA}_{\beta}\left(\subseteq \mathrm{CA}_{\alpha}\right)$ denotes the class of $\alpha$-neat reducts of $\mathrm{CA}_{\beta} \mathrm{s}$. The last class is studied extensively in the chapter [20] of [1] as a key notion in the representation theory of cylindric algebras. $\mathbf{S}$ denotes the operation of forming subalgebras and $\mathbf{P}$ denotes the operation of forming direct products. For any ordinal $\alpha, \mathrm{Cs}_{\alpha}$ denotes the class of cylindric set algebras of dimension $\alpha$ whose top elements are $\alpha$-dimensional cartesian spaces and $\mathrm{Gs}_{\alpha}$ denotes the class of generalized cylindric set algebras of dimension $\alpha$, whose top elements are generalized $\alpha$-dimensional cartesian spaces. An $\alpha$-dimensional cartesian space is a set of the form ${ }^{\alpha} U$ ( $U$ a non-empty set) and a generalized $\alpha$-dimensional cartesian space is a disjoint union of $\alpha$ dimensional cartesian spaces. By definition $\mathrm{RCA}_{\alpha}=\mathbf{S P C s}$ and it is known (and indeed not hard to show that) $\mathrm{RCA}_{\alpha}=\mathbf{I} \mathrm{Gs}_{\alpha}$ where $\mathbf{I}$ is the operation of forming isomorphic images. 
In cylindric-polyadic algebras of dimension $\alpha$ ( $\alpha$ an infinite ordinal) studied in [8], units are unions of cartesian spaces that are not necessarily disjoint. We assume familiarity with the basics of duality theory of Boolean algebras with operators BAOs, like atom structures and complex algebras. A more than an adequate reference is [12, Chapter 2]. Throughout the paper, unless otherwise indicated, we fix $2<n<\omega$.

\section{Layout}

- In $\S 2$ we recall the needed basic concepts to be used in the sequel.

- In $\S 3$ we prove negative results on OTT for $L_{n}$ algebraically by proving that infintely mjany varities of $\mathrm{CA}_{n}$ s are not atom-canonical (to be defined below).

- In $\S 4$ we prove positive results on OTT for $L_{n}$ and a multitude of algebraizable versions of $L_{\omega, \omega}$.

\section{Some basics}

We fix the notation, in the process recalling some basic needed definitions:

DeFinition 2.1. Let $\alpha$ be an ordinal and $\lambda$ be a cardinal.

(1) A weak space of dimension $\alpha$ is a set $V$ of the form $\left\{s \in{ }^{\alpha} U: \mid\{i \in\right.$ $\left.\left.\alpha: s_{i} \neq p_{i}\right\} \mid<\omega\right\}$ where $U$ is a non-empty set and $p \in{ }^{\alpha} U$. We denote $V$ by ${ }^{\alpha} U^{(p)}$. We write $\mathrm{Gws}_{\alpha}$ short hand for the class of generalized weak set algebras as defined in [10, Definition 3.1.2, item (iv)]. By definition $\mathrm{Gws}_{\alpha}=$ $\mathbf{S P W s _ { \alpha }}$, where $\mathrm{Ws}_{\alpha}$ denotes the class of weak set algebra of dimension $\alpha$. The top elements of $\mathrm{Gws}_{\alpha} \mathrm{s}$ are generalized weak spaces of dimension $\alpha$; these are disjoint unions of weak spaces of the same dimension. Plainly when $\alpha<\omega, \mathrm{Ws}_{\alpha}=\mathrm{Cs}_{\alpha}$ and $\mathrm{Gws}_{\alpha}=\mathrm{Gs}_{\alpha}$, in which case we use the notation $\mathrm{Cs}_{\alpha}$ and $\mathrm{Gs}_{\alpha}$.

Fix $\mathfrak{A} \in \mathrm{RCA}_{\alpha}$.

(2) Let $\mathbf{K} \in\left\{\mathrm{Gs}_{\alpha}, \mathrm{Gws}_{\alpha}\right\}$. If $\mathbf{X}=\left(X_{i}: i<\lambda\right)$ is family of subsets of $\mathfrak{A}$, we say that $\mathbf{X}$ is omitted with respect to $\mathbf{K}$ if there exist in $\mathfrak{C} \in \mathbf{K}_{\alpha}$, and 
an isomorphism $f: \mathfrak{A} \rightarrow \mathfrak{C}$ such that $\bigcap f\left(X_{i}\right)=\emptyset$ for all $i<\lambda$. When we want to stress the role of $f$, we say that $\mathbf{X}$ is omitted in $\mathfrak{C}$ via $f$.

(3) If $X \subseteq \mathfrak{A}$ and $\prod X=0$, then we refer to $X$ as a non-principal type of $\mathfrak{A}$.

(4) If $\mathbf{K} \in\left\{\mathrm{Gs}_{\alpha}, \mathrm{Gws}_{\alpha}\right\}, \mathfrak{A}$ is atomic and the non-principal type consisting of co-atoms (a co-atom is the complement of an atom) omitted in $\mathfrak{C} \in \mathbf{K}$ via $f$, then we say that $\mathfrak{C}$ is a complete representation of $\mathfrak{A}$ via $f$ or simply a complete representation of $\mathfrak{A}$, and that $\mathfrak{A}$ is completely representable with respect to $\mathbf{K}$.

Let $\mathbf{K} \in\left\{\mathrm{Gs}_{\alpha}, \mathrm{Gws}_{\alpha}\right\}$. It is known that an atomic $\mathfrak{A} \in \mathrm{CA}_{\alpha}$ is completely representable with respect to $\mathbf{K}$ via $f \Longleftrightarrow$ there exists $\mathfrak{C} \in \mathbf{K}$ such that for all $X \subseteq \mathfrak{A}, f\left(\sum X\right)=\bigcup_{x \in X} f(x)$, whenever $\sum X$ exists in $\mathfrak{A}$, hence the term complete representation. We note that in the last part (after the equivalence) atomicity is redundant, cf. [11].

For some time we fix $2<n<\omega$. The subtle phenomena of complete representability is closely related to the algebraic notion of atom-canonicity of (certain supervarieties of) $\mathrm{RCA}_{n}$ (like $\mathbf{S N r}_{n} \mathrm{CA}_{m}$ for $2<n<m<\omega$ ), and to the metalogical property of omitting types in $n$-variable fragments of first order logic [19, Theorems 3.1.1, 3.1.2, p. 211, Theorems 3.2.8, 3.2.9, 3.2.10], when non-principal types are omitted with respect to (relativized) semantics.

Atom-canonicity is an important persistence property in various modal logics, that applies to the class of their modal algebras; for example the variety $\mathrm{RCA}_{n}$ viewed as the class of modal algebras of the (modal formalism) of $L_{n}$ is not atom-canonical, because applying the complex algebra operator to countable atom structures of $\mathrm{RCA}_{n} \mathrm{~s}$, can give non-representable $\mathrm{CA}_{n} \mathrm{~s}$, more succintly, $\mathfrak{C m}\left(\operatorname{AtRCA}_{n}\right) \nsubseteq \mathrm{RCA}_{n}$. The term algebra on any such atom structure At say, cannot be completely representable, for a complete representation of $\mathfrak{T} \mathfrak{m A t}$ (the term algebra) induces a representation of $\mathfrak{C} \mathfrak{m} \mathbf{A t}$. This implies that OTT fails for $L_{n}$ as indicated in the introduction when $n=l$. That OTT fails for $L_{n}$ in the stronger sense indicated also in the introduction when $n<l<\omega$, follows from the fact that for all $2<n \leq l<\omega$, there exists a countable $\mathfrak{A} \in \mathrm{RCA}_{n} \cap \mathrm{Nr}_{n} \mathrm{CA}_{l}$ that is not completely representable. The last statement is proved in [2]. We start by showing that infinitely many varieties of $\mathrm{CA}_{n} s$ (containing and including $\mathrm{RCA}_{n}$ ) are not 
atom-canonical. This will imply that OTT fails strongly but in a different way; the OTT fails for $L_{n}$ with respect to so-called clique guarded semantics [13] which is a form of generalized semantics. Here the class of models allowed to omit non-principal types is broadened considerably. Models can be only $n+3-$ flat a notion to be defined below. To get an idea of the how much broadening the permissable models is occuring here; for $2<n<m<l \leq \omega$, the notion of $l$ - flatness is not finitely axiomatizable over the notion of $m$-flatness in a precise sense given in theorem 3.9 below, and that ordinary countable models coincide with $\omega$-flat models. We show that even one-non principal type in a complete and countable $L_{n}$ theory may not be omitted in any $n+k$-flat model when $k \geq 3$.

\section{Non-atom-canonicity of $\mathrm{SNr}_{n} \mathrm{CA}_{n+k}$ for $k \geq 3$ and failure of OTT with respect to clique-guarded semantics}

For sequences $f, g$ having the same domain an ordinal $\alpha$ say, and $i \in \operatorname{dom} f$, we write $f \equiv_{i} g \Longleftrightarrow f$ and $g$ agree off of $i$, that is to say $f(x)=g(x)$ for all $x \in \operatorname{dom}(f) \sim\{i\}$.

Definition 3.1. Let $2<n<\omega$ and assume that $\mathfrak{A} \in \mathrm{CA}_{n}$ is atomic.

(1) An $n$-dimensional atomic network on an $\mathfrak{A}$ is a map $N:{ }^{n} \Delta \rightarrow$ At $\mathfrak{A}$, where $\Delta$ is a non-empty set of nodes, denoted by $\operatorname{nodes}(N)$, satisfying the following consistency conditions for all $i<j<n$ :

- If $\bar{x} \in{ }^{n} \operatorname{nodes}(N)$ then $N(\bar{x}) \leq \mathrm{d}_{i j} \Longleftrightarrow x_{i}=x_{j}$,

- If $\bar{x}, \bar{y} \in{ }^{n} \operatorname{nodes}(N), i<n$ and $x \equiv_{i} y$, then $N(\bar{x}) \leq \mathrm{c}_{i} N(\bar{y})$,

(2) Assume that $m, k \leq \omega$. The atomic game $G_{k}^{m}\left(\right.$ Ata) , or simply $G_{k}^{m}$, is the game played on atomic networks of $\mathfrak{A}$ using $m$ nodes, each node only once, so that any node being used is not alllowed to be reused; and having $k$ rounds [13, Definition 3.3.2], where $\forall$ is offered only one move, namely, a cylindrifier move: Suppose that we are at round $t>0$. Then $\forall$ picks a previously played network $N_{t}\left(\operatorname{nodes}\left(N_{t}\right) \subseteq m\right), i<n, a \in$ At $\mathfrak{A}$, $x \in{ }^{n} \operatorname{nodes}\left(N_{t}\right)$, such that $N_{t}(\bar{x}) \leq \mathrm{c}_{i} a$. For her response, $\exists$ has to deliver a network $M$ such that $\operatorname{nodes}(M) \subseteq m, M \equiv_{i} N$, and there is $\bar{y} \in{ }^{n} \operatorname{nodes}(M)$ that satisfies $\bar{y} \equiv_{i} \bar{x}$ and $M(\bar{y})=a$, cf. [12, Definition 12.5(2)] for the notation $M \equiv_{i} N$. 
(3) We write $G_{k}($ At $\mathfrak{A})$, or simply $G_{k}$, for $G_{k}^{m}($ At $\mathfrak{A})$ if $m \geq \omega$.

(4) The $\omega$-rounded game $\mathbf{G}^{m}($ At $\mathfrak{A})$ or simply $\mathbf{G}^{m}$ is like the game $G_{\omega}^{m}$ (At $\left.\mathfrak{A}\right)$ except that $\forall$ has the option to reuse the $m$ nodes in play.

For BAOs, $\mathfrak{A}$ and $\mathfrak{B}$ say, having the same signature, we say that $\mathfrak{A}$ is dense in $\mathfrak{B}$ if $\mathfrak{A} \subseteq \mathfrak{B}$ and for all non-zero $b \in \mathfrak{B}$, there is a non-zero $a \in A$ such that $a \leq b$. An atom structure will be denoted by At. An atom structure $\mathbf{A t}$ has the signature of $\mathrm{CA}_{\alpha}, \alpha$ an ordinal, if $\mathfrak{C m A t}$ has the signature of $\mathrm{CA}_{\alpha}$.

Definition 3.2. Let $\mathrm{V}$ be a completely additive variety of BAOs. Then $\mathrm{V}$ is atom-canonical if whenever $\mathfrak{A} \in \mathrm{V}$ and $\mathfrak{A}$ is atomic, then $\mathfrak{C} \mathfrak{m A t} \mathfrak{A} \in \mathrm{V}$. The Dedekind-MacNeille completion of $\mathfrak{A} \in \mathrm{V}$, is the unique (up to isomorphisms that fix $\mathfrak{A}$ pointwise) complete $\mathfrak{B}$ such that $\mathfrak{A} \subseteq \mathfrak{B}$ and $\mathfrak{A}$ is dense in $\mathfrak{B}$.

From now on fix $2<n<\omega$. If $\mathfrak{A} \in \mathrm{CA}_{n}$ is atomic, then $\mathfrak{C m A t} \mathfrak{A}$ is the Dedekind-MacNeille completion of $\mathfrak{A}$. If $\mathfrak{A} \in \mathrm{CA}_{n}$, then its atom structure will be denoted by At $\mathfrak{A}$ with domain the set of atoms of $\mathfrak{A}$ denoted by At $\mathfrak{A}$.

Lemma 3.3. Let $2<n<m<\omega$ and assume that $\mathfrak{A} \in \mathrm{CA}_{n}$ is atomic. If $\mathfrak{A} \in \mathbf{S}_{c} \mathrm{Nr}_{n} \mathrm{CA}_{m}$, then $\exists$ has a winning strategy in $\mathbf{G}^{m}($ At $\mathfrak{A})$. In particular, If $\mathfrak{A}$ is finite and $\forall$ has a winning strategy in $\mathbf{G}_{\omega}^{m}(\mathrm{At} \mathfrak{A})$, then $\mathfrak{A} \notin \mathbf{S N r}_{n} \mathrm{CA}_{m}$.

Proof: [23, Lemma 4.3].

In the next theorem 3.5, we show non-atom canonicity of the varieties $\mathrm{SNr}_{n} \mathrm{CA}_{n+k}$ for $k \geq 3$. The gist of the idea is a combination of the modeltheoretic techniques of Hodkinson's used in [15] conjuncted with a blow up and blur construction in the sense of [2]. The idea of a 'a blow up and blur' construction is simple, but powerful and subtle. We give the general idea. One starts with a finite algebra $\mathfrak{D} \in \mathrm{CA}_{n}$, blowing its atom structure, by splitting one or more of its atoms into infinitely many thereby obtaining a new infinite atom structure, call it At, such that $\mathfrak{D}$ embeds into $\mathfrak{C} \mathbf{m} A \mathbf{t}$. If $\mathfrak{D}$ is not representable, or even has only finite representations (representations on finite bases) and $\mathfrak{T} \mathfrak{m A t}$ happens to be representable, then the DedekindMacNeille completion $\mathfrak{C} \mathfrak{m A t}$ of $\mathfrak{T} \mathfrak{m A t}$ will not be representable, because a representation of the infinite algebra $\mathfrak{C} \mathbf{m} \mathbf{A t}$ necessarily has an infinite base, inducing an infinite representation of $\mathfrak{D}$, since $\mathfrak{D}$ embeds in $\mathfrak{C} \mathbf{m} \mathbf{A t}$ and $\mathrm{RCA}_{n}$ is a variety. So one thereby obtains a weakly representable atom 
structure At, that is not strongly representable. But this same idea can also be applied to the varieties $\mathrm{V}_{k}=\mathbf{S N r}_{n} \mathrm{CA}_{n+k}$ for $k>1$, approximating $\mathrm{RCA}_{n}$. One blows up and blur a finite algebra $\mathfrak{D}$ outside the (larger) $\mathrm{V}_{\mathrm{k}}$ (when $k<\omega$ ), thereby obtaining a weakly representable atom structure At, such $\mathfrak{C} \mathfrak{m} A t$ is outside $V_{k}$ because $\mathfrak{D}$ embeds into $\mathfrak{C m A t}$. If for some $k_{0}>1$, the atom structure At obtained after blowing up and blurring the finite algebra that is outside $V_{k_{0}}$ is representable, it will readily follow that $\mathrm{V}_{\mathrm{k}}$, for all $k \geq k_{0}$ is not atom-canonical. The term blur refers to the fact that the algebraic structure of $\mathfrak{D}$ is blurred at the level of $\mathfrak{T} \mathfrak{m} \mathbf{A t}$, it does not embed into $\mathfrak{T} \mathbf{m A t}$ prohibiting its representability, but it it is not blurred on the 'global level' of $\mathfrak{C m A t}$, in the sense that $\mathfrak{D}$ embeds into $\mathfrak{C m} \mathbf{A t}$.

One might be tempted to think that our next result can be obtained by 'lifting somehow' to higher dimensions the construction for RAs proving that $\mathbf{S} \mathfrak{R a C A} A_{k}, k \geq 6$ is not atom-canonical proved in [12] using a blow up and blur construction for relation algebras. In [12], an representable atomic relation algebra $\mathfrak{R}$, whose Dedekind-MacNeille completion is outside $\mathbf{S} \mathfrak{R a C A} \mathrm{C}_{6}$, is constructed. But this cannot be done with the lifting construction in [18] as it stands, for given an atomic $\mathfrak{R} \in \mathrm{RA}$, it does not necessarily embed in the Ra reduct of the atomic $\mathrm{CA}_{n}$ constructed from the $\mathfrak{R}$ as described in op. cit if $n \geq 6$. It can only be done for $n=3$. We briefly review the blow up and blur construction in $[12,17.32,17.34,17.36]$ for relation algebras proving that $\mathbf{S} \mathfrak{R a C A}$, for $k \geq 6$ is not atom canonical. We need some preparation. Let $2 \leq n \leq \omega$ and $r \leq \omega$. Let $\mathfrak{R}$ be an atomic relation algebra. Then the $r$-rounded game $G_{r}^{n}($ At $\Re)$ [12, Definition 12.24] is the (usual) atomic game played on networks of an atomic relation algebra $\mathfrak{R}$ using $n$ nodes.

Let $L$ be a relational signature. Let $\mathrm{G}$ (the greens) and $\mathrm{R}$ (the reds) be $L$ structures and $p, r \leq \omega$. The game $\operatorname{EF}_{r}^{p}(\mathrm{G}, \mathrm{R})$, defined in $[12$, Definition 16.1.2], is an Ehrenfeucht-Fraïssé forth 'pebble game' with $r$ rounds and $p$ pairs of pebbles. In $[12,16.2]$, a relation algebra rainbow atom structure is associated for relational structures $\mathrm{G}$ and R. We denote by $\mathbf{R}_{A, B}$ the (full) complex algebra over this atom structure. The Rainbow Theorem [12, Theorem 16.5] states that: If $\mathrm{G}, \mathrm{R}$ are relational structures and $p, r \leq \omega$, then $\exists$ has a winning strategy in $G_{1+r}^{2+p}\left(\mathbf{R}_{\mathrm{G}, \mathfrak{R}}\right) \Longleftrightarrow$ she has a winning strategy in $\mathrm{EF}_{r}^{p}(\mathrm{G}, \mathrm{R})$.

For $5 \leq l<\omega, \mathrm{RA}_{l}$ is the class of relation algebras whose canonical extensions have an $l$-dimensional relational basis [12, Definition 12.30]. $\mathrm{RA}_{l}$ is a variety containing properly the variety $\mathbf{S} \mathfrak{R a C A} A_{l}$. Furthermore, $\exists$ has 
a winning strategy in $G_{\omega}^{n}($ At $\Re) \Longrightarrow \Re \in \mathrm{RA}_{l}$, cf. [12, Proposition 12.31] and [12, Remark 15.13]. We now show:

Theorem 3.4. For any $k \geq 6$, the varieties $\mathrm{RA}_{k}$ and $\mathbf{S} \mathfrak{R a C A} A_{k}$ are not atom-canonical.

Proof: We follow the notation in [12, lemmas 17.32, 17.34, 17.35, 17.36] with the sole exception that we denote by $m$ (instead of $\mathbf{K}_{m}$ ) the complete irreflexive graph on $m$ defined the obvious way; that is we identify this graph with its set of vertices. Fix $2<n<m<\omega$. Let $\mathfrak{R}=\mathbf{R}_{m, n}$. Then by the rainbow theorem $\forall$ has a winning strategy in $G_{m+1}^{m+2}($ At $\Re)$, since it clealy has a winning strategy in the Ehrenfeucht-Fraïssé game $\operatorname{EF}_{m}^{m}(m, n)$ because $m$ is 'longer' than $n$. Then $\Re \notin \mathrm{RA}_{m+2}$ by [12, Propsition 12.25 , Theorem $13.46(4) \Longleftrightarrow(5)]$, so $\mathfrak{R} \notin \mathbf{S} \mathfrak{R a C A}{ }_{m+2}$. Next one 'splits' every red atom to $\omega$-many copies obtaining the infinite atomic countable (term) relation algebra denoted in op.cit by $\mathcal{T}$, with atom structure $\alpha$, cf. [12, item (4) top of p. 532]. Then $\mathfrak{C} \mathfrak{m} \alpha \notin \mathbf{S} \mathfrak{R a C A}{ }_{m+2}$ because $\mathfrak{R}$ embeds into $\mathfrak{C} m \alpha$ by mapping every red to the join of its copies, and $\mathbf{S} \mathfrak{R a C A} \mathbf{A}_{m+2}$ is closed under S. Finally, one (completely) represents (the canonical extension of) $\mathcal{T}$ like in [12]. By taking $m=4$ and $n=3$ the required follows.

We next blow up and blur a finite rainbow $\mathrm{CA}_{n}(2<n<\omega)$. The proof, otherwise, is presented in a model-theoretic framework as done in [15], where it is proved that $\mathrm{RCA}_{n}$ is not atom-canonical. We briefly review rainbow constructions for CAs [11, 13]. Fix $2<n<\omega$. Given relational structures $\mathrm{G}$ (the greens) and $\mathrm{R}$ (the reds) the rainbow atom structure of a $\mathrm{CA}_{n}$ consists of equivalence classes of surjective maps $a: n \rightarrow \Delta$, where $\Delta$ is a coloured graph. A coloured graph is a complete graph labelled by the rainbow colours, the greens $\mathrm{g} \in \mathrm{G}$, reds $\mathrm{r} \in \mathrm{R}$, and whites; and some $n-1$ tuples are labelled by 'shades of yellow'. In coloured graphs certain triangles are not allowed for example all green triangles are forbidden. Some (but not all) of the red triples are forbidden. cf. [11, 4.3.3]. The equivalence relation relates two such maps $\Longleftrightarrow$ they essentially define the same graph $[11,4.3 .4]$. We let $[a]$ denote the equivalence class containing $a$. The accessibilty (binary relations) corresponding to cylindric operations are like in [11]. Special coloured graphs typically used by $\forall$ during implementing his winning strategy are called cones: Let $i \in \mathrm{G}$, and let $M$ be a coloured graph consisting of $n$ nodes $x_{0}, \ldots, x_{n-2}, z$. We call $M$ an $i$ - cone if $M\left(x_{0}, z\right)=\mathrm{g}_{0}^{i}$ and for every $1 \leq j \leq n-2, M\left(x_{j}, z\right)=\mathrm{g}_{j}$, and no other 
edge of $M$ is coloured green. $\left(x_{0}, \ldots, x_{n-2}\right)$ is called the base of the cone, $z$ the apex of the cone and $i$ the tint of the cone. For $2<n<\omega$, we use the graph version of the games $G_{\omega}^{m}(\beta)$ and $\mathbf{G}^{m}(\beta)$ where $\beta$ is a $\mathbf{C A}_{n}$ rainbow atom structure, cf. $[11,4.3 .3]$. The (complex) rainbow algebra based on $\mathrm{G}$ and $\mathrm{R}$ is denoted by $\mathfrak{A}_{\mathrm{G}, \mathrm{R}}$. The dimension $n$ will always be clear from context. For relation algebras the relation algebra $\mathbf{R}_{4.3}$ was blown up and blurred, now we blow up and blur $\mathrm{CA}_{n+1, n}$

TheOREM 3.5. Let $2<n<\omega$. Then there exists $\mathfrak{B} \in \mathrm{Cs}_{n}$ such that

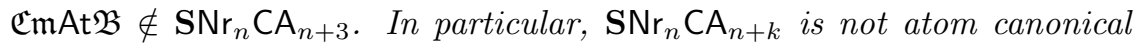
for all $k \geq 3$

Proof: We finish off with the second part modulo the first. Then we prove the first part. We have $\mathfrak{B} \in \mathrm{RCA}_{n}=\bigcap_{m>0} \mathrm{SNr}_{n} \mathrm{CA}_{n+m}$ but $\mathfrak{C m A t} \mathfrak{B} \notin$ $\mathrm{SNr}_{n} \mathrm{CA}_{n+k}$ for all $k \geq 3$.

The proof of the first part is divided to three parts:

(a) Blowing up and blurring a finite rainbow algebra: Take the finite CA rainbow algebra $\mathfrak{D}$ as defined in [13] where the reds $\mathrm{R}$ is the complete irreflexive graph $n$, and the greens are $\mathrm{G}=\left\{\mathrm{g}_{i}: 1 \leq i<n-1\right\} \cup\left\{\mathrm{g}_{0}^{i}\right.$ : $1 \leq i \leq n+1\}$, endowed with the polyadic operations. Denote $\mathfrak{D}$ by $\mathrm{CA}_{n+1, n}$ and for the sake of brevity, denote its finite atom structure by $\mathbf{A t}_{f}$; so that $\mathbf{A t}_{f}=\operatorname{At}\left(\mathrm{CA}_{n+1, n}\right)$. One then replaces the red colours of the finite rainbow algebra of $\mathrm{CA}_{n+1, n}$ each by infinitely many reds (getting their superscripts from $\omega$ ), obtaining this way a weakly representable atom structure At. The resulting atom structure after 'splitting the reds', namely, At, is like the weakly but not strongly representable atom structure of the atomic, countable and simple algebra $\mathfrak{A}$ constructed in [15], the sole difference is that we have $n+1$ greens and not infinitely many as is the case in [15]. We denote our algebra also by $\mathfrak{A}$. No confusion is likely to ensue. We will go further by showing that $\mathfrak{C m A t A} \notin \mathbf{S N r}_{n} \mathrm{CA}_{n+3}$. The rainbow signature [13, Definition 3.6.9] $L$ now consists of $\mathrm{g}_{i}: 1 \leq i<n-1$, $\mathrm{g}_{0}^{i}: 1 \leq i \leq n+1, \mathrm{w}_{i}: i<n-1, \mathrm{r}_{k l}^{t}: k<l<n, t \in \omega$, binary relations, and $n-1$ ary relations $\mathrm{y}_{S}, S \subseteq n+1$. There is a shade of red $\rho$; the latter is a binary relation that is outside the rainbow signature, but it labels coloured graphs during a 'rainbow game'. $\exists$ can win the rainbow $\omega$-rounded game and build an $n$-homogeneous model $M$ by using $\rho$ when she is forced a red; [15, Proposition 2.6, Lemma 2.7]. From now on, forget about $\rho$; having done its task as a colour to (weakly) represent $\mathfrak{A}$, it will 
play no further role. Having $\mathrm{M}$ at hand, one constructs two atomic $n-$ dimensional set algebras based on $\mathrm{M}$, sharing the same atom structure and having the same top element. The atoms of each will be the set of coloured graphs, seeing as how, quoting Hodkinson [15] such coloured graphs are 'literally indivisible'. Now $L_{n}$ and $L_{\infty, \omega}^{n}$ are taken in the rainbow signature (without $\rho$ ). Continuing like in op.cit, deleting the one available red shade, set $W=\left\{\bar{a} \in{ }^{n} \mathrm{M}: \mathrm{M}=\left(\bigwedge_{i<j<n} \neg \rho\left(x_{i}, x_{j}\right)\right)(\bar{a})\right\}$, and for $\phi \in L_{\infty, \omega}^{n}$, let $\phi^{W}=\{s \in W: \mathrm{M}=\phi[s]\}$. Here $W$ is the set of all $n$-ary assignments in ${ }^{n} \mathrm{M}$, that have no edge labelled by $\rho$. We note that $\rho$ is used by $\exists$ infinitely many times during the game forming a 'red clique' in $M$ [15]. Let $\mathfrak{A}$ be the relativized set algebra with domain $\left\{\varphi^{W}: \varphi\right.$ a first-order $L_{n}$-formula $\}$ and unit $W$, endowed with the usual concrete operations read off the connectives. Classical semantics for $L_{n}$ rainbow formulas and their semantics by relativizing to $W$ coincide [15, Proposition 3.13] but not with respect to $L_{\infty, \omega}^{n}$ rainbow formulas. This depends essentially on [15, Lemma 3.10], which is the heart and soul of the proof in [15], and for what matters this proof. The referred to lemma says that any permutation $\chi$ of $\omega \cup\{\rho\}$, $\Theta^{\chi}$ as defined in [15, Definitions 3.9, 3.10] is an $n$ back-and-forth system induced by any permutation of $\omega \cup\{\rho\}$. Let $\chi$ be such a permutation. Thee system $\Theta^{\chi}$ consists of isomorphisms between coloured graphs such that the superscripts of reds are 're-shuffled along' $\chi$ in such a way that rainbow red labels are permuted $\rho$ is replaced by a red rainbow colour, and all other colours are preserved. One uses such $n$-back-and-forth systems mapping a tuple $\bar{b} \in{ }^{n} \mathrm{M} \sim W$ to a tuple $\bar{c} \in W$ preserving any formula in the rainbow signature not containing the non-red symbols that are 'moved' by the system, so if $\bar{b} \in{ }^{n} \mathrm{M}$ refutes the $L_{n}$ rainbow formula $\phi$, then there is a $\bar{c} \in W$ refuting $\phi$, as well. The rainbow algebra $\mathfrak{A}$ is then isomorphic to cylindric set algebra having top element ${ }^{n} \mathrm{M}$, so $\mathfrak{A}$ is simple, in fact it can be shown that even its diagonal free reduct is simple. Let $\mathfrak{E}=\left\{\phi^{W}: \phi \in L_{\infty, \omega}^{n}\right\}$ [15, Definition 4.1] with the operations defined like on $\mathfrak{A}$ the usual way. $\mathfrak{C m A t}$ is complete and, so like in [15, Lemma 5.3] we have an isomorphism from $\mathfrak{C}$ At to $\mathfrak{E}$ defined via $X \mapsto \bigcup X$. We have At $\mathfrak{A}=$ At $\mathfrak{T} \mathfrak{m}($ At $\mathfrak{A})=$ At (where $\mathfrak{T} \mathfrak{m}($ At $\mathfrak{A})$ denotes the subalgebra of $\mathfrak{C} \mathfrak{m} A t \mathfrak{A}$ generated by the atoms; the term algebra) and $\mathfrak{T} \mathfrak{m} A t \mathfrak{A} \subseteq \mathfrak{A}$, hence $\mathfrak{T} \mathfrak{m} A t \mathfrak{A}$ is representable. The atoms of $\mathfrak{A}, \mathfrak{T} \mathfrak{m A t} \mathfrak{A}$ and $\mathfrak{C m A t \mathfrak { A }}=\mathfrak{C m A t}$ are the coloured graphs whose edges are not labelled by $\rho$. These atoms are uniquely determined (syntactically) by MCA formulas in the rainbow signature of At as in [15, Definition 
4.3]. The expression blow up and blur is an indicative term introduced in [2]. Blowing up means splitting the atoms of a finite algebra; in our context $\mathrm{CA}_{n+1, n}$ each into infinitely many obtaining a new atom structure denoted above by At. Blurring, means that the algebraic structure of $\mathrm{CA}_{n+1, n}$ is blurred in $\mathfrak{T} \mathfrak{m A t}$, its algebraic structure is disorganized or distorted in such a way that it does not embed into $\mathfrak{T} \mathbf{m} A t$. Nevertheless, it reapperas in the Dedekind-MacNeille completion of $\mathfrak{T} \mathfrak{m A t}$, namely, in $\mathfrak{C} \mathbf{m A t}$ as we shall see in a moment; $\mathrm{CA}_{n+1, n}$ embeds into $\mathfrak{C} \mathbf{m} \mathbf{A t}$ by mapping every splitted 'red atom' to the suprema of the subatoms into which it was split. This sprema exists because (the Boolean reduct of) $\mathfrak{C} \mathfrak{m} \mathbf{A t}$ is a complete algebra, which is not the case with $\mathfrak{T} \mathfrak{m A t}$. The last is not complete,

(b) Embedding $\mathrm{CA}_{n+1, n}$ into the complex algebra $\mathfrak{C}$ At: Now to embed $\mathrm{CA}_{n+1, n}$ into $\mathfrak{C} \mathbf{m} \mathbf{A t}=\mathfrak{C} \mathfrak{m} A t \mathfrak{A}$, we need some preparing to do. To start with, we Identify $r$ with $r^{0}$, so that we consider that $\mathbf{A t}_{f} \subseteq \mathbf{A t}$. Let $\mathrm{CRG}_{f}$ be the class of coulored graphs on $\mathbf{A t}_{f}$ and CRG be the class of coloured graph on At. By the above identification, we can assume that $\mathrm{CRG}_{f} \subseteq \mathrm{CRG}$. Write $M_{a}$ for the atom that is the (equivalence class of the) surjection $a: n \rightarrow M, M \in$ CGR. Here we identify $a$ with $[a]$; no harm will ensue. We define the (equivalence) relation $\sim$ on At by $M_{b} \sim N_{a}$, $(M, N \in \mathrm{CGR})$

- $a(i)=a(j) \Longleftrightarrow b(i)=b(j)$,

- $M_{a}(a(i), a(j))=\mathrm{r}^{l} \Longleftrightarrow N_{b}(b(i), b(j))=\mathrm{r}^{k}$, for some $l, k \in \omega$,

- $M_{a}(a(i), a(j))=N_{b}(b(i), b(j))$, if they are not red,

- $M_{a}\left(a\left(k_{0}\right), \ldots, a\left(k_{n-2}\right)\right)=N_{b}\left(b\left(k_{0}\right), \ldots, b\left(k_{n-2}\right)\right)$, whenever defined.

We say that $M_{a}$ is a copy of $N_{b}$ if $M_{a} \sim N_{b}$ (by symmetry $N_{b}$ is a copy of $M_{a}$.) Indeed, the relation 'copy of' is an equivalence relation on At. An atom $M_{a}$ is called a red atom, if $M$ has at least one red edge. Any red atom plainly has $\omega$-many copies (including itself); furthermore (as is the case with splitting arguments) all such copies are cylindrically equivalent, in the sense that, if $N_{a} \sim M_{b}$ with one (equivalently both) red, with $a: n \rightarrow N$ and $b: n \rightarrow M$, then we can assume that $\operatorname{nodes}(N)=\operatorname{nodes}(M)$ and that for all $i<n, a\left\lceil n \sim\{i\}=b\left\lceil n \sim\{i\}\right.\right.$. In $\mathfrak{C m A t}$, we write $M_{a}$ for $\left\{M_{a}\right\}$ and we denote suprema taken in $\mathfrak{C} \mathbf{m A t}$, possibly finite, by $\sum$. If $N_{b}$ is a red copy of $M_{a}$, then we may denote $N_{b}$ by $M_{a}^{(j)}(j \in \omega)$. Note that a red atom $M_{a}$ has $\omega$ many copies forming a countable (infinite) set 
$\left\{M_{a}^{(j)}: j \in \omega\right\}$ of red graphs. If $M_{a}$ is a red atom, then by $\sum_{j} M_{a}^{(j)}$ we understand the infinite sum of its copies evaluated in $\mathfrak{C m A t}$. If $M_{a}$ is not red, then it has only one copy, namely, itself. Now we define the map $\Theta$ from $\mathrm{CA}_{n+1, n}=\mathfrak{C m} \mathbf{A t} t_{f}$ to $\mathfrak{C m} \mathbf{A t}$, by $\Theta(X)=\bigcup_{x \in \mathbf{A t}_{\mathbf{f}}} \Theta(x)\left(X \subseteq \mathbf{A t}_{f}\right)$, by specifing first its values on $\mathrm{At}_{f}$, via $M_{a} \mapsto \sum_{j} M_{a}^{(j)}$; each atom maps to the suprema of its copies. If $M_{a}$ is not red, then by $\sum_{j} M_{a}^{(j)}$, we understand $M_{a}$. This map is well-defined because $\mathfrak{C} \mathbf{m}$ At is complete. We check that $f$ is an injective homomorphim. Injectivity follows from $M_{a} \leq f\left(M_{a}\right)$, hence $f(x) \neq 0$ for every atom $x \in \operatorname{At}\left(\mathrm{CA}_{n+1, n}\right)$. Now we check presevation of operations. The Boolean join is obvious.

- For complementation: It suffices to check preservation of complementation 'at atoms' of $\mathbf{A t}_{f}$. So let $M_{a} \in \mathbf{A t}_{f}$ with $a: n \rightarrow M$, $M \in \mathrm{CGR}_{\mathrm{f}} \subseteq \mathrm{CGR}$. Then:

$$
\begin{aligned}
\Theta\left(\sim M_{a}\right) & =\Theta\left(\bigcup_{[b] \neq[a]} M_{b}\right)=\bigcup_{[b] \neq[a]} f\left(M_{b}\right)=\bigcup_{[b] \neq[a]} \sum_{j} M_{b}^{(j)} \\
& =\bigcup_{[b] \neq[a]} \sim \sum_{j}\left[\sim\left(M_{a}\right)^{(j)}\right]=\bigcup_{[b] \neq[a]} \sim \sum_{j}\left[\left(\sim M_{b}\right)^{j}\right] \\
& =\bigcup_{[b] \neq[a]} \bigwedge_{j} M_{b}^{(j)}=\bigwedge_{j} \bigcup_{[b] \neq[a]} M_{b}^{(j)}=\bigwedge_{j}\left(\sim M_{a}\right)^{j}=\sim\left(\sum M_{a}^{j}\right) \\
& =\sim \Theta(a) .
\end{aligned}
$$

- Diagonal elements. Let $l<k<n$. Then:

$$
\begin{aligned}
M_{x} \leq f\left(\mathbf{d}_{l k}^{\mathfrak{C m} \mathbf{A} \mathbf{t}_{\mathbf{f}}}\right) & \Longleftrightarrow M_{x} \leq \sum_{j} \bigcup_{a_{l}=a_{k}} M_{a}^{(j)} \\
& \Longleftrightarrow M_{x} \leq \bigcup_{a_{l}=a_{k}} \sum_{j} M_{a}^{(j)} \\
& \Longleftrightarrow M_{x}=M_{a}^{(j)} \text { for some } a: n \rightarrow M \text { such that } \\
& \Longleftrightarrow M_{x} \in \mathrm{d}_{l k}^{\mathfrak{C m} \mathbf{A t}} .
\end{aligned}
$$

- Cylindrifiers. Let $i<n$. By additivity of cylindrifiers, we restrict our attention to atoms $M_{a} \in \mathbf{A t}_{\mathbf{f}}$ with $a: n \rightarrow M$, and $M \in \mathrm{CRG}_{\mathrm{f}} \subseteq \mathrm{CRG}$. 
Then:

$$
\begin{aligned}
f\left(\mathrm{c}_{i}^{\mathfrak{C} \mathbf{m} \mathbf{A t}_{\mathbf{f}}} M_{a}\right) & =f\left(\bigcup_{[c] \equiv_{i}[a]} M_{c}\right)=\bigcup_{[c] \equiv_{i}[a]} f\left(M_{c}\right) \\
& =\bigcup_{[c] \equiv_{i}[a]} \sum_{j} M_{c}^{(j)}=\sum_{j} \bigcup_{[c] \equiv_{i}[a]} M_{c}^{(j)} \\
& =\sum_{j} c_{i}^{\mathfrak{C} \mathfrak{A} \mathbf{A t}} M_{a}^{(j)}=c_{i}^{\mathfrak{C} \mathfrak{A} \mathbf{A t}}\left(\sum_{j} M_{a}^{(j)}\right) \\
& =c_{i}^{\mathfrak{C} \mathbf{A t}} f\left(M_{a}\right) .
\end{aligned}
$$

We have proved that $\mathbf{C A}_{n+1, n}$ embeds into $\mathfrak{C m} \mathbf{A t}$, so that it is not blurred at the level of the last complex algebra.

(c) $\forall \mathbf{s}$ winning strategy in $\mathbf{G}^{n+3}\left(\mathrm{AtCA}_{n+1, n}\right)$ : It is straightforward to show that, like in the relation algebra case that $\forall$ has a winning strategy in the Ehrenfeucht-Fraïssé forth private game played between $\exists$ and $\forall$ on the complete irreflexive graphs $n+1$ and $n$, namely, in $\mathrm{EF}_{n+1}^{n+1}(n+1, n)$ (using $n+1$ pebble pairs in $n+1$ rounds). This game lifts to a graph game [11, pp.841] on $\mathbf{A t}_{f}$ which in this case is equivalent to the graph version of $\mathbf{G}^{n+3}$, but here $\forall$ does not need to re-use pebbles, so that the game is actually $G^{n+3}$ but of course it ends after only finitely many rounds. $\forall$ lifts his winning strategy from the private Ehrenfeucht-Fraïssé forth game, to the graph game on $\mathbf{A t}_{f}=\operatorname{At}\left(\mathrm{CA}_{n+1, n}\right)$ using the standard rainbow strategy [11]. He bombards $\exists$ with cones having the same base with green tints, demanding that $\exists$ delivers a red label each time for the succesive appexes of the cones he plays. It is not hard to show that he will need two more nodes in the graph game to win. Thus by lemma 3.3, $\mathrm{CA}_{n+1, n} \notin \mathrm{SNr}_{n} \mathrm{CA}_{n+3}$.

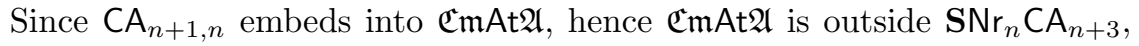
too.

Remark 3.6. One can describe $\mathrm{CA}_{n+1, n}$ differently as a subalgebra of the algebra $\mathfrak{C}$ in [15, Defnition 5.1] as foillows. Let $Z$ be the finite subsignature of $L$ obtained by deletng all $r_{j k}^{i}$ for $i>0$ but keeping $r_{j k}^{0}$. For each $Z_{\infty \omega}^{n}$ formulu $\phi$, Define the $L_{\infty \omega}$ formula $\left.\hat{(} \phi\right)$ to be the result of replacing each subformula $r_{j k}^{0}(x, y)$ in $\phi$ by $\bigvee_{i \in \omega} r_{j k}^{i}(x, y)$. It is clearly a finite subagebra of $\mathfrak{C}$ with atoms $\hat{\alpha}^{W}$ where $\alpha$ is an MCA $Z^{n}$ formula as defined in [15]. 
Corollary 3.7. There are infinitely many subvarieties of $\mathrm{CA}_{n}$ containing $\mathrm{RCA}_{n}$ that are not atom-canonical.

Proof: It is known that for any pair of ordinals $\alpha<\beta, \mathbf{S N r}_{\alpha} \mathrm{CA}_{\beta}$ is a variety, and that for $k \geq 1$ an $2<n<\omega, \mathbf{S N r}_{n} \mathrm{CA}_{n+k+1} \subsetneq \mathbf{S N r}_{n} \mathrm{CA}_{n+k}$ [12, Chapter 15]

Using the previous algebraic result on non atom canonicity, we adress algebraically a version of the omitting types theorems in the framework of the clique guarded $n$-variable fragments of first order logic. We define the notion of clique guarded semantics.

Definition 3.8. Let $2<n \leq m<\omega$. Let $\mathrm{M}$ be the base of a relativized representation of $\mathfrak{A} \in \mathrm{CA}_{n}$ witnessed by an injective homomorphism $f$ : $\mathfrak{A} \rightarrow \wp(V)$, where $V \subseteq{ }^{n} \mathrm{M}$ and $\bigcup_{s \in V} \mathrm{rng}(s)=\mathrm{M}$. We write $\mathrm{M}=a(s)$ for $s \in f(a)$. Let $\mathfrak{L}(\mathfrak{A})^{m}$ be the first order signature using $m$ variables and one $n$-ary relation symbol for each element in $A$. Let $\mathfrak{L}(\mathfrak{A})_{\infty, \omega}^{m}$ be the infinitary extension of $\mathfrak{L}(\mathfrak{A})^{m}$ allowing infinite conjunctions. Then an $n$-clique is a set $C \subseteq \mathrm{M}$ such $\left(a_{1}, \ldots, a_{n}\right) \in V=1^{\mathrm{M}}$ for distinct $a_{1}, \ldots, a_{n} \in C$.

Let $\mathrm{C}^{m}(\mathrm{M})=\left\{s \in{ }^{m} \mathrm{M}: \operatorname{rng}(s)\right.$ is an $n$-clique $\} . \mathrm{C}^{m}(\mathrm{M})$ is called the $n$-Gaifman hypergraph of $\mathrm{M}$, with the $n$-hyperedge relation $1^{\mathrm{M}}$.

The clique guarded semantics $\models_{c}$ are defined inductively. For atomic formulas and Boolean connectives they are defined like the classical case and for existential quantifiers (cylindrifiers) they are defined as follows: for $\bar{s} \in{ }^{m} \mathrm{M}, i<m, \mathrm{M},\left.\bar{s}\right|_{{ }_{c}} \exists x_{i} \phi \Longleftrightarrow$ there is a $\bar{t} \in \mathrm{C}^{m}(\mathrm{M}), \bar{t} \equiv_{i} \bar{s}$ such that $\mathrm{M}, \bar{t} \models \phi$.

(1) We say that $M$ is an $m$-square representation of $\mathfrak{A}$, if for all $\bar{s} \in$ $\mathrm{C}^{m}(\mathrm{M}), a \in \mathfrak{A}, i<n$, and injective map $l: n \rightarrow m$, whenever $\mathrm{M} \models$ $\mathrm{c}_{i} a\left(s_{l(0)}, \ldots, s_{l(n-1)}\right)$, then there is a $\bar{t} \in \mathrm{C}^{m}(\mathrm{M})$ with $\bar{t} \equiv_{i} \bar{s}$, and $\mathrm{M} \models$ $a\left(t_{l(0)}, \ldots, t_{l(n-1)}\right) . \mathrm{M}$ is a complete $m$-square representation of $\mathfrak{A}$ via $f$, or simply a complete representation of $\mathfrak{A}$ if $f\left(\sum X\right)=\bigcup_{x \in X} f(x)$, for all $X \subseteq \mathfrak{A}$ for which $\sum X$ exists. (Like in the classical case this is equivalent to that $\mathfrak{A}$ is atomic and that $\left.\bigcup_{x \in \text { At } \mathfrak{A}} f(x)=1^{\mathrm{M}}\right)$.

(2) We say that $\mathrm{M}$ is an (infinitary) $m$-flat representation of $\mathfrak{A}$ if it is $m$ square and for all $\phi \in\left(\mathfrak{L}(\mathfrak{A})_{\infty, \omega}^{m}\right) \mathfrak{L}(\mathfrak{A})^{m}$, for all $\bar{s} \in \mathrm{C}^{m}(\mathrm{M})$, for all distinct $i, j<m, \mathrm{M} \models_{c}\left[\exists x_{i} \exists x_{j} \phi \longleftrightarrow \exists x_{j} \exists x_{i} \phi\right](\bar{s})$. Complete representability is defined like for squareness. 
The proof of the following lemma can be distilled from its RA analogue [12, Theorem 13.20], by reformulating deep concepts originally introduced by Hirsch and Hodkinson for RAs in the CA context. cf. [12, Definitions $12.1,12.9,12.10,12.25$, Propositions 12.25, 12.27].

Theorem 3.9. [12, Theorems 13.45, 13.36]. Assume that $2<n<m<\omega$ and let $\mathfrak{A} \in \mathrm{CA}_{n}$. Then $\mathfrak{A} \in \mathbf{S N r}_{n} \mathrm{CA}_{m} \Longleftrightarrow \mathfrak{A}$ has an infinitary m-flat representation $\Longleftrightarrow \mathfrak{A}$ has an $m$-flat representation. In particular, the variety of algebras having $m+1$-flat representations is not finitely axiomatizable over the variety of algebras having $m$-flat representations.

Proof: We give (more than) a glimpse of the ideas used. We prove first that the existence of $m$-flat representations, implies the existence of $m^{-}$ dilations. Let $\mathrm{M}$ be an $m$-flat representation of $\mathfrak{A}$. We show that $\mathfrak{A} \subseteq$ $\mathrm{Nr}_{n} \mathfrak{D}$, for some $\mathfrak{D} \in \mathrm{CA}_{m}$, For $\phi \in \mathfrak{L}(\mathfrak{A})^{m}$ (as defined above), let $\phi^{\mathrm{M}}=$ $\left\{\bar{a} \in \mathrm{C}^{m}(\mathrm{M}): \mathrm{M}=_{c} \phi(\bar{a})\right\}$, where $\mathrm{C}^{m}(\mathrm{M})$ is the $n$-Gaifman hypergraph. Let $\mathfrak{D}$ be the algebra with universe $\left\{\phi^{M}: \phi \in \mathfrak{L}(\mathfrak{A})^{m}\right\}$ and with cylindric operations induced by the $n$-clique-guarded (flat) semantics. Recall that for $r \in \mathfrak{A}$, and $\bar{x} \in \mathrm{C}^{m}(\mathrm{M})$, we identify $r$ with the formula it defines in $\mathfrak{L}(\mathfrak{A})^{m}$, and we write $r(\bar{x})^{\mathrm{M}} \Longleftrightarrow \mathrm{M}, \bar{x} \models_{c} r$. Then certainly $\mathfrak{D}$ is a subalgebra of the $\mathrm{Crs}_{m}$ (the class of algebras whose units are arbitrary sets of $m$-ary sequences) with domain $\wp\left(\mathrm{C}^{m}(\mathrm{M})\right)$, so $\mathfrak{D} \in \mathrm{Crs}_{m}$ with unit $1^{\mathfrak{D}}=\mathrm{C}^{m}(\mathrm{M})$. Since $\mathrm{M}$ is $m$-flat, then cylindrifiers in $\mathfrak{D}$ commute, and so $\mathfrak{D} \in \mathrm{CA}_{m}$. Now define $\theta: \mathfrak{A} \rightarrow \mathfrak{D}$, via $r \mapsto r(\bar{x})^{\mathrm{M}}$. Then exactly like in the proof of [12, Theorem 13.20], $\theta$ is a neat embedding, that is, $\theta(\mathfrak{A}) \subseteq \mathrm{Nr}_{n} \mathfrak{D}$. It is straightforward to check that $\theta$ is a homomorphism. We show that $\theta$ is injective. Let $r \in A$ be non-zero. Then $\mathrm{M}$ is a relativized representation, so there is $\bar{a} \in M$ with $r(\bar{a})$, hence $\bar{a}$ is a clique in $\mathrm{M}$, and so $M \models r(\bar{x})(\bar{a})$, and $\bar{a} \in \theta(r)$, proving the required. $\mathrm{M}$ itself might not be infinitary $m$-flat, but one can build an infinitary $m$-flat representation of $\mathfrak{A}$, whose base is an $\omega$-saturated model of the consistent first order theory, stipulating the existence of an $m$-flat representation, cf. [12, Proposition 13.17, Theorem 13.46 items (6) and (7)]. The inverse implication (existence of $m$-dilations $\Longrightarrow$ existence of $m$-flat represenations) is harder. One constructs from the given $m$-dilation, an $m$-dimensional hyperbasis (redeined to adapt to $\mathrm{CA}_{n} \mathrm{~s}$ without too much difficulty) from which the required $m$ - relativized representation is built. This can be done in a step-by step manner treating the hyperbasis as a 'saturated set of mosaics', cf. [12, Proposition 12.37]. 
The last part follows from $[13, \S 15.1-3]$ where it is proved that $\mathbf{S} \mathrm{Nr}_{n} \mathbf{C A} \mathbf{A}_{m+1}$ is not finitely axiomatizable over $\mathbf{S N r}_{n} \mathrm{CA}_{m}$.

Lemma 3.10. Let $2<n<m<\omega$, and $\mathfrak{A} \in \mathrm{CA}_{n}$ be an atomic algebra. Then $\mathfrak{A}$ has a complete $m$-square representation $\Longleftrightarrow \exists$ has a winning strategy in $G_{\omega}^{m}(\mathrm{At} \mathfrak{A})$.

Proof: [22, Lemma 5.8].

Corollary 3.11. There exists $\mathfrak{A} \in \mathrm{Cs}_{n}$ such that $\mathfrak{C} \mathfrak{m} A t \mathfrak{A}$ does not have an $n+3$-square representation.

Proof: This follows from the previous Lemma, together with the proof of (c) in Theorem 3.5 by observing that $\forall$ has a winning strategy in $G_{\omega}^{n+3} \mathrm{CA}_{n+1, n}$ (in finitely many rounds of course) without the need to reuse nodes. The game $\mathbf{G}^{m}$ is stronger than what is really needed.

LEMMA 3.12. if $\mathfrak{A} \in \mathrm{CA}_{n}$ has a complete $m$-flat representation, then $\mathfrak{A}$ is

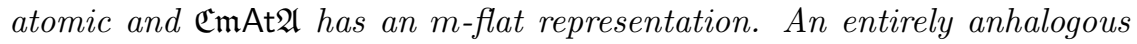
result holds by replacing $m$-flat by $m$-square.

Proof: Atomicity is like the classical case [11]. Now let $f: \mathfrak{A} \rightarrow \wp(V)$ be a complete $m$-flat representation $\mathfrak{A}$ with $V \subseteq{ }^{n} \mathrm{M}$ where $\mathrm{M}$ is the base of the representation, so that $\mathrm{M}=\bigcup_{s \in V} \operatorname{rng}(s)$. For $a \in \mathfrak{C m A t a}$, let $a \downarrow=\{x \in$ AtA $: x \leq a\}$. Define $g: \mathfrak{C m A t A} \rightarrow \wp(V)$ by $g(a)=\bigcup_{x \in \downarrow a} f(x)$. Then $g$ is a complete $m$-flat representation of $\mathfrak{C} \mathfrak{m A t} \mathfrak{A}$ with base M.

For an $L_{n}$ theory $T, \mathfrak{F m}_{T}$, denotes the Tarski-Lindenbaum quotient RCA $_{n}$ corresponding to $T$ where the quoitent modulo $T$ is defined semantically. Given an $L_{n}$ theory $T$ and $m>n$, by an $m$-flat model of $T$, we understand an $m$ - flat representation of $\mathfrak{F m}_{T}$ when $m<\omega$, and an ordinary representation of $\mathfrak{F m}_{T}$ if $m$ is infinite. An atomic $L_{n}$ theory $T$ is one for which $\mathfrak{F m}_{T}$ is atomic. A co-atom of $T$ is a formula $\phi$ such that $(\neg \phi)_{T}$ is an atom in $\mathfrak{F m}_{T}$.

Corollary 3.13. There is a countable, atomic and complete $L_{n}$ theory $T$ such that the non-principal type consisting of co-atoms cannot be omitted in an $n+3$-square, a fortiori $n+3$-flat model.

Proof: Let $\mathfrak{A} \in \mathrm{Cs}_{n}$ be countable (and simple) such that its DedekindMacNeille completion does not have an $n+3$-square representation. This 
$\mathfrak{A}$ exists by Theorem 3.5. By [10, $\S 4.3]$, we can (and will) assume that $\mathfrak{A}=\mathfrak{F m}_{T}$ for a countable, atomic theory $L_{n}$ theory $T$. Let $\Gamma$ be the $n$-type consisting of co-atoms of $T$. Then $\Gamma$ is a non principal type that cannot be omitted in any $n+3$-square model, for if $\mathrm{M}$ is an $n+3$-square model omitting $\Gamma$, then $\mathrm{M}$ would be the base of a complete $n+3$-square representation of $\mathfrak{A}$, giving, by Lemma 3.12, representation of $\mathfrak{C} \mathfrak{m} A t \mathfrak{A}$, which is impossible.

There exists a countable, complete and atomic $L_{n}$ first order theory $T$ in a signature $L$ such that the type $\Gamma$ consisting of co-atoms in the cylindric Tarski-Lindenbaum quotient algebra $\mathfrak{F m}_{T}$ is realizable in every $m$-square model, but $\Gamma$ cannot be isolated using $\leq l$ variables, where $n \leq l<m \leq \omega$. A co-atom of $\mathfrak{F m}_{T}$ is the negation of an atom in $\mathfrak{F m}_{T}$, that is to say, is an element of the form $\Psi / \equiv_{T}$, where $\Psi / \equiv_{T}=\left(\neg \phi / \equiv_{T}\right)=\sim\left(\phi / \equiv_{T}\right)$ and $\phi / \equiv_{T}$ is an atom in $\mathfrak{F m}_{T}$ (for $L$-fomrulas, $\phi$ and $\psi$ ). Here the quotient algebra $\mathfrak{F m}_{T}$ is formed relative to the congruence relaton of semantical equivalence moduol $T$; for formulas $\phi$ and $\theta$ in the signature $L, \phi \equiv_{T} \theta$ $\Longleftrightarrow T \models \phi \longleftrightarrow \theta$. An $m$-square model of $T$ is an $m$-square represenation of $\mathfrak{F m}_{T}$. The statement $\Psi(l, m)$, short for Vaught's Theorem (VT) fails at (the parameters) $l$ and $m$. Let $\mathrm{VT}(l, m)$ stand for $\mathrm{VT}$ holds at $l$ and $m$, so that by definition $\Psi(l, m) \Longleftrightarrow \neg \mathrm{VT}(l, m)$. We also include $l=\omega$ in the equation by defining $\operatorname{VT}(\omega, \omega)$ as $\mathrm{VT}$ holds for $L_{\omega, \omega}$ : Atomic countable first order theories have atomic countable models. It is well known that $\operatorname{VT}(\omega, \omega)$ is a direct consequence of the Orey-Henkin OTT. Let $2<n \leq l<m \leq \omega$. Consider the statemens $\Psi(l, m)$ and $\operatorname{VT}(l, m)=\neg \Psi(l, m)$ as defined in the introduction. Recall that $\operatorname{VT}(\omega, \omega)$ is just Vaught's theorem, namely, countable atomic theories have atomic countable models. For $2<n \leq l<m \leq \omega$ and $l=m=\omega$, it is likely and plausible that $(* *): \mathrm{VT}(l, m) \Longleftrightarrow l=m=\omega$. In other words: Vaught's theorem holds only in the limiting case when $l \rightarrow \infty$ and $m=\omega$ and not 'before'. We give sufficient condition for $(* *)$ to happen.

Theorem 3.14. For $2<n<\omega$ and $n \leq l<\omega, \Psi(n, n+3)$ and $\Psi(l, \omega)$ hold. Furthermore, if for each $n<m<\omega$, there exists a finite relation algebra $\mathfrak{R}_{m}$ having $m-1$ strong blur and no $m$-dimensional relational basis, then (**) above for VT holds.

Proof: We start by the last part. Let $\mathfrak{R}_{m}$ be as in the hypothesis with strong $m-1$-blur $(J, E)$ and $m$-dimensional relational basis. We 'blow 
up and blur' $\mathfrak{R}_{m}$ in place of the Maddux algebra $\mathfrak{E}_{k}(2,3)$ blown up and blurred in [2, Lemma 5.1], where $k<\omega$ is the number of non-identity atoms and $k$ depends recursively on $l$, giving the desired strong $l$-blurness, cf. [2, Lemmata 4.2, 4.3]. Now take $\mathfrak{A}=\mathfrak{B b}_{n}\left(\mathfrak{R}_{m}, J, E\right)$ the term algebra obtained after blowing up and blurring $\mathfrak{R}$ to a weakly representable atom structure [2]. Then $\mathfrak{A} \in \mathrm{RCA}_{n} \cap \mathrm{Nr}_{n} \mathrm{CA}_{l}$ but $\mathfrak{A}$ has no complete $m$-square representation. For if it did, then a complete $m$-square representation of an atomic $\mathfrak{B} \in \mathrm{CA}_{n}$ induces an $m$-square representation of $\mathfrak{C} \mathfrak{m A t} \mathfrak{B}$. But $\mathfrak{C m A t} \mathfrak{A}$ does not have an $m$-square representation, because $\mathfrak{R}$ does not have an $m$-dimensional relational basis, and $\mathfrak{R} \subseteq \mathfrak{R a C m A t} \mathfrak{A}$. So an $m$-square

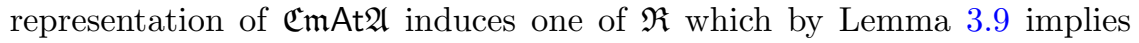
that $\mathfrak{R}$ has no $m$-dimensional relational basis, a contradiction.

We prove $\Psi(m-1, m)$, hence the required, namely, $(* *)$. By $[10, \S 4.3]$, we can (and will) assume that $\mathfrak{A}=\mathfrak{F m}_{T}$ for a countable, simple and atomic theory $L_{n}$ theory $T$. Let $\Gamma$ be the $n$-type consisting of co-atoms of $T$. Then $\Gamma$ is realizable in every $m$-square model, for if $\mathrm{M}$ is an $m$-square model omitting $\Gamma$, then $\mathrm{M}$ would be the base of a complete $m$-square representation of $\mathfrak{A}$, and so by Theorem $3.9 \mathfrak{A} \in \mathbf{S}_{c} \mathrm{Nr}_{n} \mathrm{D}_{m}$ which is impossible. Suppose for contradiction that $\phi$ is an $m-1$ witness, so that $T \models \phi \rightarrow \alpha$, for all $\alpha \in \Gamma$, where recall that $\Gamma$ is the set of coatoms. Then since $\mathfrak{A}$ is simple, we can assume without loss that $\mathfrak{A}$ is a set algebra with base $M$ say. Let $\mathrm{M}=\left(M, R_{i}\right)_{i \in \omega}$ be the corresponding model (in a relational signature) to this set algebra in the sense of $[10, \S 4.3]$. Let $\phi^{\mathrm{M}}$ denote the set of all assignments satisfying $\phi$ in $\mathrm{M}$. We have $\mathrm{M} \models T$ and $\phi^{\mathrm{M}} \in \mathfrak{A}$, because $\mathfrak{A} \in \mathrm{Nr}_{n} \mathrm{CA}_{m-1}$. But $T=\exists x \phi$, hence $\phi^{\mathrm{M}} \neq 0$, from which it follows that $\phi^{\mathrm{M}}$ must intersect an atom $\alpha \in \mathfrak{A}$ (recall that the latter is atomic). Let $\psi$ be the formula, such that $\psi^{\mathrm{M}}=\alpha$. Then it cannot be the case that $T \models \phi \rightarrow \neg \psi$, hence $\phi$ is not a witness, contradiction and we are done. Finally, $\Psi(n, n+3)$ and $\Psi(l, \omega)(n \leq l<\omega)$ follow from Corollary 3.13 and $[2]$.

Corollary 3.15. There exists an atomic $\mathcal{T} \in$ RRA and an atomic $\mathfrak{A} \in$ $\mathrm{RCA}_{n}$ such that their Dedekind-MacNeille completions do not embed into their canonical extensions.

Proof: We prove the CA case only. The RA case is entirely analagous. Since $\mathrm{RCA}_{n}$ is canonical [10] and $\mathfrak{A} \in \mathrm{RCA}_{n}$, then its canonical extension $\mathfrak{A}^{+} \in \mathrm{RCA}_{n}$. But $\mathfrak{C m A t \mathfrak { A }} \notin \mathrm{RCA}$, so it does not embed into $\mathfrak{A}^{+}$, because $\mathrm{RCA}_{n}$ is a variety, a fortiori closed under $\mathbf{S}$. 
Algebraically, so-called persistence properties refer to closure of a variety $\mathrm{V}$ under passage from a given algebra $\mathfrak{A} \in \mathrm{V}$ to some 'larger' algebra $\mathfrak{A}^{*}$. Atom-canonicity is concerned with closure under forming DedekindMacNeille completions. Atom-canonicity, implies the algebraic property of single-persistence which in turn corresponds in modal logic to the notion of a formula being di-persistent. A formula is di-persistent if whenever it is valid in some general discrete frame $(\mathfrak{F}, P)$, that is, $P$ contains all singletions, then is valid in the Kripke frame $\mathfrak{F}[4, \S 5.6]$. Sometimes DedekindMacNeille completions, investigated for cylindric algebras by Monk, are referred to as minimal completions, the name suggesting that DedekindMacNeille completion of an algebra $\mathfrak{A}$ is the 'smallest' in the sense that it embeds into other any completion of $\mathfrak{A}$. Here by a completion we understand any complete algebra containing $\mathfrak{A}$. Canonicity, which is the most prominent persistence property in modal logic, the 'large algebra' $\mathfrak{A}^{*}$ is the canonical embedding algebra (or perfect) extension of $\mathfrak{A}$, a complex algebra based on the ultrafilter frame of $\mathfrak{A}$, in symbols Uf $A$, whose underlying set is the set of all Boolean ultrafilters of $\mathfrak{A}$. This is another completion of $\mathfrak{A}$. The Dedekind-MacNeille completion of a BAO and its canonical extension coincide $\Longleftrightarrow \mathfrak{A}$ is finite. By the last result formulated in Corollary 3.15 the term minimal is misleading. A minimal completion of $\mathfrak{A} \in \mathrm{RCA}_{n} \mathrm{~s}$, namely $\mathfrak{C} \mathfrak{m} A$ t $\mathfrak{A}$, may not embed into its canonical extension $\mathfrak{A}^{+}=\mathfrak{C} \mathfrak{m} U \mathbf{f} \mathfrak{A}$.

Canonicity corresponds to the notion of a formula being dpersistent [4, Definition 5.65, Proposition 5.85]. A modal formula in $L_{n}$ is canonical if it is validated in the canonical frame of every normal modal logic containing $\phi[4$, Definition 4.30]. Algebraically, $\phi$ is canonical $\Longleftrightarrow \phi$ translates to an equation in the signature of $\mathrm{RCA}_{n}$ that is preserved under canonical extensions. An example of formulas that are both di-persistent and canonical (d-persistent) are the so-called very simple Sahlqvist formulas [4, Theorem 5.90] which are, as the name suggests, instances of Sahlqvist formulas [12, Definition 3.51].

Sahlqvist formulas are a certain kind of modal formula with remarkable properties. The Sahlqvist correspondence theorem states that every Sahlqvist formula corresponds to a first order definable class of Kripke frames. Sahlqvist's definition characterizes a decidable set of modal formulas with first-order correspondents. Since it is undecidable, by Chagrova's theorem, whether an arbitrary modal formula has a first-order correspondent [4, Theorem 3.56], there are formulas with first-order frame conditions that are not Sahlqvist. But this is not the end of the story, for it might 
be the case that every modal formula with a first order correspondant is equivalent to a Sahlqvist one, which is not the case [4, Example 3.57]. The reader is referred to [4] and [12, 2.7] for more on aspects of duality for BAOs and in particular for Sahlvist axiomatizability in general. By the dualiity theory betwem BAOs and multimodal logic, Sahlqvist formulas in the latter transfrm to Sahlqvist equations in modal algebras. A variety V of BAOs is Sahlqvist if it can be axiomatized by Sahlvist equations.

Theorem 3.16. For any $2<n<m \leq \omega$ the variety $\mathbf{S N r}_{n} \mathrm{CA}_{m}$ is not Sahlqvist. Conversely, for any pair of infinite ordinals $\alpha<\beta$, the varieties $\mathrm{SNr}_{\alpha} \mathrm{PA}_{\beta}$ and $\mathbf{S N r}_{\alpha} \mathrm{PEA}_{\beta}$ are Sahlqvist, and is closed under Dedekind-MacNeille completions.

Proof: Let $\alpha<\beta$ be infinite ordinals. Then $\mathrm{SNr}_{\alpha} \mathrm{PA}_{\beta}=\mathrm{Nr}_{\alpha} \mathrm{PA}_{\beta}=\mathrm{PA}_{\alpha}$, cf. the remark before [10, Theorem 5.4.17]. The last is axiomatized by positive equations [10, Definition 5.4.1] which are Sahlqvist. Applying [25] we are done. The PEA case is entirely analogous using the axiomatization in the aforementioned definition.

Let $2<n<\omega$. We approach the modal version of $L_{n}$ without equality, namely, $\mathbf{S 5}^{n}$. The corresponding class of modal algebras is the variety $\mathrm{RDf}_{n}$ of diagonal free $\mathrm{RCA}_{n} s$ [10]. Let $\mathfrak{R \mathfrak { d } _ { d f }}$ denote 'diagonal free reduct'.

Lemma 3.17. Let $2<n<\omega$. If $\mathfrak{A} \in \mathrm{CA}_{n}$ is such that $\mathfrak{R} \mathfrak{d}_{d f} \mathfrak{A} \in \mathrm{RDf}_{n}$, and $\mathfrak{A}$ is generated by $\{x \in \mathfrak{A}: \Delta x \neq n\}$ (with other CA operations) using infinite intersections, then $\mathfrak{A} \in \mathrm{RCA}_{n}$.

Proof: Easily follows from [10, Lemma 5.1.50, Theorem 5.1.51]. Assume that $\mathfrak{A} \in \mathrm{CA}_{n}, \mathfrak{R} \mathfrak{d}_{d f} \mathfrak{A}$ is a set algebra (of dimension $n$ ) with base $U$, and $R \subseteq U \times U$ are as in the hypothesis of [10, Theorem 5.1.49]. Let $E=\left\{x \in A:\left(\forall x, y \in{ }^{n} U\right)(\forall i<n)\left(x_{i} R y_{i} \Longrightarrow(x \in X \Longleftrightarrow y \in X)\right)\right\}$. Then $\{x \in \mathfrak{A}: \Delta x \neq n\} \subseteq E$ and $E \in \mathrm{CA}_{n}$ is closed under infinite intersections. The required follows.

TheOREM 3.18. For $2<n<\omega, \mathrm{RDf}_{n}$ is not atom-canonical, hence not Sahlqvist.

Proof: It is enough to show that $\mathfrak{C} \mathfrak{m} A t \mathfrak{A}$, where $\mathfrak{A}$ is constructed in Theorem 3.5 is generated by elements whose dimension sets have cardinality $<n$ using infinite unions, for in this case $\mathfrak{R} \mathfrak{d}_{d f} A$ will be atomic, countable and representable, but having no complete representation. Indeed, 
by Lemma 3.17 and Theorem 3.5, $\mathfrak{R} \mathfrak{d}_{d f} \mathfrak{C m A t \mathfrak { A }}=\mathfrak{C} \mathfrak{m A t} \mathfrak{R} \mathfrak{d}_{d f} \mathfrak{A}$ will not be representable. We show that for any rainbow atom $[a], a: n \rightarrow \Gamma$, $\Gamma$ a coloured graph, that $[a]=\prod_{i<n} c_{i}[a]$. Clearly $\leq$ holds. Assume that $b: n \rightarrow \Delta, \Delta$ a coloured graph, and $[a] \neq[b]$. We show that $[b] \notin \prod_{i<n} c_{i}[a]$ by which we will be done. Because $a$ is not equivalent to $b$, we have one of two possibilities; either $(\exists i, j<n)(\Delta(b(i), b(j) \neq$ $\Gamma(a(i), a(j))$ or $\left(\exists i_{1}, \ldots, i_{n-1}<n\right)\left(\Delta\left(b_{i_{1}}, \ldots, b_{i_{n-1}}\right) \neq \Gamma\left(a_{i_{1}}, \ldots, a_{i_{n-1}}\right)\right)$. Assume the first possibility: Choose $k \notin\{i, j\}$. This is possible because $n>2$. Assume for contradiction that $[b] \in c_{k}[a]$. Then $(\forall i, j \in$ $n \backslash\{k\})(\Delta(b(i), b(j))=\Gamma(a(i) a(j)))$. By assumption and the choice of $k$, $(\exists i, j \in n \backslash k)(\Delta(b(i), b(j)) \neq \Gamma(a(i), a(j)))$, contradiction. For the second possibility, one chooses $k \notin\left\{i_{1}, \ldots i_{n-1}\right\}$ and proceeds like the first case deriving an analogous contradiction.

$\mathbf{K}^{n}$ is the logic of $n$-ary product frames, of the form $\left(W_{i}, R_{i}\right)_{i<n}$ where for each $i<n, R_{i}$ is any any relation on $W_{i}$. On the other hand, $\mathbf{S} 5^{n}$ can be regarded as the logic of $n$-ary product frames of the form $\left(W_{i}, R_{i}\right)_{i<n}$ such that for each $i<n, R_{i}$ is an equivalence relation. It is known that logics between $\mathbf{K}^{n}$ and $\mathbf{S} 5^{n}$ are quite complicated, cf. [16] for a detailed overview. Theorem 3.19 to be proved in a moment adds to their complexity.

It is known that modal languages can come to grips with a strong fragment of second order logic. Modal formulas translate to second order formulas, their correspondants on frames. Some of these formulas can be genuinely second order; they are not equivalent to first order formulas. An example is the McKinsey formula: $\square \diamond p \rightarrow \diamond \square p$. This can be proved by showing that its correspondant violates the downward Löwenheim- Skolem Theorem. The next proposition bears on the last two issues. For a class $\mathbf{L}$ of frames, let $\mathfrak{L}(\mathbf{L})$ be the class of modal formulas valid in $\mathbf{L}$. It is difficult to find explicity (necessarily) infinite axiomatizations for $\mathbf{S} 5^{n}$ as well:

Theorem 3.19. Let $2<n<\omega$. There is no axiomatization of $\mathbf{S} 5^{n}$ with formulas having first order correspondence. For any canonical logic $\mathfrak{L}$ between $\mathbf{K}^{n}$ and $\mathbf{S} 5^{n}$, it is undecidable to tell whether a finite frame is a frame for $\mathfrak{L}, \mathfrak{L}$ cannot be finitely axiomatized in kth order logic (for any finite $k$ ), and $\mathfrak{L}$ cannot be axiomatized by canonical formulas, a fortiori Sahlqvist formulas.

Proof: Let $\mathbf{L}$ be the class of square frames for $\mathbf{S} 5^{n}$. Then $\mathfrak{L}(\mathbf{L})=\mathbf{S} 5^{n}$ $[16$, p. 192]. But the class of frames $\mathfrak{F}$ valid in $\mathfrak{L}(\mathbf{L})$ coincides with the 
class of strongly representable $\mathrm{Df}_{n}$ atom structures which is not elementary as proved in [5]. This gives the first required result for $\mathbf{S 5}^{n}$. With lemma 3.17 at our disposal, a slightly different proof can be easily distilled from the construction adressing CAs in [13] or [14]. We adopt the construction in the former reference, using the Monk-like $\mathrm{CA}_{n} \mathrm{~S} \mathfrak{M}(\Gamma), \Gamma$ a graph, as defined in [13, Top of p.78]. For a graph $\mathfrak{G}$, let $\chi(\mathfrak{G})$ denote it chromatic number. Then it is proved in op.cit that for any graph $\Gamma, \mathfrak{M}(\Gamma) \in \mathrm{RCA}_{n}$ $\Longleftrightarrow \chi(\Gamma)=\infty$. By lemma 3.17, $\mathfrak{R d}_{d f} \mathfrak{M}(\Gamma) \in \operatorname{RDf}_{n} \Longleftrightarrow \chi(\Gamma)=\infty$, because $\mathfrak{M}(\Gamma)$ is generated by the set $\{x \in \mathfrak{M}(\Gamma): \Delta x \neq n\}$ using infinite unions.

Now we adopt the argument in [13]. Using Erdos' probabalistic graphs [7], for each finite $\kappa$, there is a finite graph $G_{\kappa}$ with $\chi\left(G_{\kappa}\right)>\kappa$ and with no cycles of length $<\kappa$. Let $\Gamma_{\kappa}$ be the disjoint union of the $G_{l}$ for $l>\kappa$. Then $\chi\left(\Gamma_{\kappa}\right)=\infty$, and so $\mathfrak{R} \mathfrak{d}_{d f} \mathfrak{M}\left(\Gamma_{\kappa}\right)$ is representable. Now let $\Gamma$ be a nonprincipal ultraproduct $\Pi_{D} \Gamma_{\kappa}$ for the $\Gamma_{\kappa} \mathrm{s}$. For $\kappa<\omega$, let $\sigma_{\kappa}$ be a first-order sentence of the signature of the graphs stating that there are no cycles of length less than $\kappa$. Then $\Gamma_{l} \models \sigma_{\kappa}$ for all $l \geq \kappa$. By Loś's Theorem, $\Gamma \models \sigma_{\kappa}$ for all $\kappa$. So $\Gamma$ has no cycles, and hence by $\chi(\Gamma) \leq 2$. Thus $\mathfrak{R} \mathfrak{d}_{d f} \mathfrak{M}(\Gamma)$ is not representable. (Observe that the the term algebra $\mathfrak{T} \mathfrak{m A t}(\mathfrak{M}(\Gamma)$ ) is representable (as a $\mathrm{CA}_{n}$ ), because the class of weakly representable atom structures is elementary [12, Theorem 2.84].) Since Sahlqvist formulas have first order correspondants, then $\mathbf{S 5}^{n}$ is not Sahlqvist. In [14], it is proved that it is undecidable to tell whether a finite frame is a frame for $\mathfrak{L}$, and this gives the non-finite axiomatizability result required as indicated in op. cit, and obviously implies undecidability. The rest follows by transferring the required results holding for $\mathbf{S} 5^{n}[5,14]$ to $\mathfrak{L}$ since $\mathbf{S} 5^{n}$ is finitely axiomatizable over $\mathfrak{L}$, and any axiomatization of $\operatorname{RDf}_{n}$ must contain infinitely many non-canonical equations.

Results involving notions like atom-canonicity, for the infinite dimensional case, are extremely rare in algebraic logic [13, Problem 3.8.3]; in fact, almost non-existent. We present a conditional result (the condition is very likely to be true). For each finite $k \geq 3$, let $\mathfrak{A}(k)$ be an atomic countable simple representable $\mathrm{CA}_{k}$ such that $\mathfrak{B}(k)=\mathfrak{C} \mathfrak{m A t} \mathfrak{A}(k) \notin \mathbf{S N r}_{k} \mathrm{CA}_{k+3}$. We know that such algebras exist by Theorem 3.5. We make the following assumption: $\left(^{*}\right)$ Assume that $\mathfrak{B}(m)$ embeds into $\mathfrak{R} \mathfrak{d}_{m} \mathfrak{B}(t)$, whenever $3 \leq m<t<\omega$. Our next theorem lifts Theorem 3.5 to the transfinite conditionally (modulo $(*))$. 
THEOREM 3.20. Assume that (*) above holds for the algebras constructed in Theorem 3.5 (or any other algebras). Then for $k \geq 3, \mathrm{SNr}_{\omega} \mathrm{CA}_{\omega+k}$ is not atom-canonical. In particular, $\mathrm{RCA}_{\omega}$ cannot be axiomatized by (a necessarily infinite schema of) Sahlqvist equations.

Proof: For each finite $k \geq 3$, let $\mathfrak{A}(k)$ and $\mathfrak{B}(k)$ be the algebras constructed in Theorem 3.5 (of dimension $k$ ) and assume further that the assumption abbreviated by $(*)$ preceding the theorem holds for the algebras constructed in op.cit. Let $\mathfrak{A}_{k}$ be an (atomic) algebra having the signature of $\mathrm{CA}_{\omega}$ such that $\mathfrak{R d}_{k} \mathfrak{A}_{k}=\mathfrak{A}(k)$. Analogously, let $\mathfrak{B}_{k}$ be an algebra having the signature of $\mathrm{CA}_{\omega}$ such that $\mathfrak{R d}_{k} \mathfrak{B}_{k}=\mathfrak{B}(k)$, and we require in addition that $\mathfrak{B}_{k}=\mathfrak{C m}\left(\right.$ At $\left.\mathfrak{A}_{k}\right)$. We use a lifting argument using ultraproducts. Let $\mathfrak{B}=\Pi_{i \in \omega \backslash 3} \mathfrak{B}_{i} / F$. It is easy to show that $\mathfrak{A}=\Pi_{i \in \omega \backslash 3} \mathfrak{A}_{i} / F \in \mathrm{RCA}_{\omega}$. Furthermore, a direct computation gives:

$$
\begin{aligned}
\mathfrak{C m A t} \mathfrak{A} & \left.=\mathfrak{C m}\left(\operatorname{At}\left[\Pi_{i \in \omega \backslash 3} \mathfrak{A}_{i} / F\right]\right)=\mathfrak{C m}\left[\Pi_{i \in \omega \backslash 3}\left(\operatorname{At}_{i}\right) / F\right)\right] \\
& =\Pi_{i \in \omega \backslash 3}\left(\mathfrak{C m}\left(\operatorname{At}_{i}\right) / F\right)=\Pi_{i \in \omega \backslash 3} \mathfrak{B}_{i} / F \\
& =\mathfrak{B} .
\end{aligned}
$$

By the same token, $\mathfrak{B} \in \mathrm{CA}_{\omega}$. Assume for contradiction that $\mathfrak{B} \in \mathbf{S N r}_{\omega} \mathrm{CA}_{\omega+3}$. Then $\mathfrak{B} \subseteq \mathfrak{N r}_{\omega} \mathfrak{C}$ for some $\mathfrak{C} \in \mathrm{CA}_{\omega+3}$. Let $3 \leq m<\omega$ and let $\lambda: m+3 \rightarrow \omega+3$ be the function defined by $\lambda(i)=i$ for $i<m$ and $\lambda(m+i)=\omega+i$ for $i<3$. Then we get $(* *): \mathfrak{R o}^{\lambda} \mathfrak{C} \in \mathrm{CA}_{m+3}$ and $\mathfrak{R} \mathfrak{d}_{m} \mathfrak{B} \subseteq \mathfrak{N r}_{m} \mathfrak{R d}{ }^{\lambda} \mathfrak{C}$. By assumption let $I_{t}: \mathfrak{B}_{m} \rightarrow \mathfrak{R} \mathfrak{d}_{m} \mathfrak{B}_{t}$ be an injective homomorphism for $3 \leq m<t<\omega$. Let $\iota(b)=\left(I_{t} b: t \geq m\right) / F$ for $b \in \mathfrak{B}_{m}$. Then $\iota$ is an injective homomorphism that embeds $\mathfrak{B}_{m}$ into $\mathfrak{R} \mathfrak{d}_{m} \mathfrak{B}$. By (**) we know that $\mathfrak{R} \mathfrak{d}_{m} \mathfrak{B} \in \mathbf{S N r}_{m} \mathrm{CA}_{m+3}$, hence $\mathfrak{B}_{m} \in \mathbf{S N r}_{m} \mathrm{CA}_{m+3}$, too. This is a contradiction, and we are done.

\section{Positive results on omitting types}

We start by recalling certain cardinals that play a key role in (positive) omitting types theorems for $L_{\omega, \omega}$. Let covK be the cardinal used in [19, Theorem 3.3.4]. The cardinal $\mathfrak{p}$ satisfies $\omega<\mathfrak{p} \leq 2^{\omega}$ and has the following property: If $\lambda<\mathfrak{p}$, and $\left(A_{i}: i<\lambda\right)$ is a family of meager subsets of a Polish space $X$ (of which Stone spaces of countable Boolean algebras are examples) then $\bigcup_{i \in \lambda} A_{i}$ is meager. For the definition and required properties of $\mathfrak{p}$, witness [9, pp. 3, 44-45, Corollary 22c]. 
It is consistent that $\omega<\mathfrak{p}<\operatorname{covK} \leq 2^{\omega}[9]$, but it is also consistent that they are equal; equality holds for example in the Cohen real model of Solovay and Cohen. Martin's axiom implies that both cardinals are the continuum. To prove the main result on positive omitting types theorems, we need the following lemma due to Shelah:

Lemma 4.1. Assume that $\lambda$ is an infinite regular cardinal. Suppose that $T$ is a first order theory, $|T| \leq \lambda$ and $\phi$ is a formula consistent with $T$, then there exist models $\mathrm{M}_{i}: i<{ }^{\lambda}$, each of cardinality $\lambda$, such that $\phi$ is satisfiable in each, and if $i(1) \neq i(2), \bar{a}_{i(l)} \in M_{i(l)}, l=1,2, \operatorname{tp}\left(\bar{a}_{l(1)}\right)=$ $\operatorname{tp}\left(\bar{a}_{l(2)}\right)$, then there are $p_{i} \subseteq \operatorname{tp}\left(\bar{a}_{l(i)}\right),\left|p_{i}\right|<\lambda$ and $p_{i} \vdash \operatorname{tp}\left(\bar{a}_{l(i)}\right)(\operatorname{tp}(\bar{a})$ denotes the complete type realized by the tuple $\bar{a}$ )

Proof: [24, Theorem 5.16, Chapter IV].

In the next theorem $n<\omega$. Furthermore the maximality condition expressed in ultrafilters (which are maximal filters) delineates the edge of an independent statement to a provable one. Considering only filters leads to an independent statement, cf. [19, Theorem 3.2.8]:

THEOREM 4.2. Let $\mu$ be a countable or regular uncountable cardinal. Let $\mathfrak{A} \in \mathbf{S}_{c} \mathrm{Nr}_{n} \mathrm{CA}_{\omega}$ be such that $|A| \leq 2^{\mu}$. Let $\lambda<2^{\mu}$ and let $\mathbf{X}=\left(X_{i}: i<\lambda\right)$ be a family of non-principal types of $\mathfrak{A}$. Then the following hold:

(1) If $\mathfrak{A} \in \mathrm{Nr}_{n} \mathrm{CA}_{\omega}$ and the $X_{i}$ s are non-principal ultrafilters, then $\mathbf{X}$ can be omitted in a $\mathrm{Gs}_{n}$. Furthrmore, the condition of maximality cannot be dispensed with,

(2) If $\mathfrak{A}$ is countable, then every subfamily of $\mathbf{X}$ of cardinality $<\mathfrak{p}$ can be omitted in a $\mathrm{Gs}_{n}$; in particular, every countable subfamily of $\mathbf{X}$ can be omitted in a $\mathrm{Gs}_{n}$, If $\mathfrak{A}$ is simple, then every subfamily of $\mathbf{X}$ of cardinlity $<$ covK can be omitted in $a \mathrm{Cs}_{n}$.

Proof: For the first item we prove the special case when $\mu \omega$. The general case follows from the fact that $(* *)$ below holds for any infinite regular cardinal. We assume that $\mathfrak{A}$ is simple (a condition that can be easily removed). We have $\prod^{\mathfrak{B}} X_{i}=0$ for all $i<\kappa$ because, $\mathfrak{A}$ is a complete subalgebra of $\mathfrak{B}$. Since $\mathfrak{B}$ is a locally finite (if not replace $\mathfrak{B}$ by $\mathfrak{S} g^{\mathfrak{B}} \mathfrak{A}$ ), we can assume that $\mathfrak{B}=\mathfrak{F m}_{T}$ for some countable consistent theory $T$. For each $i<\kappa$, let $\Gamma_{i}=\left\{\phi / T: \phi \in X_{i}\right\}$. Let $\mathbf{F}=\left(\Gamma_{j}: j<\kappa\right)$ be the 
corresponding set of types in $T$. Then each $\Gamma_{j}(j<\kappa)$ is a non-principal and complete $n$-type in $T$, because each $X_{j}$ is a maximal filter in $\mathfrak{A}=\mathfrak{N r}_{n} \mathfrak{B}$.

(**) Let $\left(\mathrm{M}_{i}: i<2^{\omega}\right)$ be a set of countable models for $T$ that overlap only on principal maximal types; these exist by lemma 4.1. Asssume for contradiction that for all $i<2^{\omega}$, there exists $\Gamma \in \mathbf{F}$, such that $\Gamma$ is realized in $\mathbf{M}_{i}$. Let $\psi: 2^{\omega} \rightarrow \wp(\mathbf{F})$, be defined by $\psi(i)=\{F \in \mathbf{F}$ : $F$ is realized in $\left.\mathrm{M}_{i}\right\}$. Then for all $i<2^{\omega}, \psi(i) \neq \emptyset$. Furthermore, for $i \neq j$, $\psi(i) \cap \psi(j)=\emptyset$, for if $F \in \psi(i) \cap \psi(j)$, then it will be realized in $\mathbf{M}_{i}$ and $\mathbf{M}_{j}$, and so it will be principal. This implies that $|\mathbf{F}|=2^{\omega}$ which is impossible. Hence we obtain a model $\mathrm{M} \models T$ omitting $\mathbf{X}$ in which $\phi$ is satisfiable. The map $f$ defined from $\mathfrak{A}=\mathfrak{F m}_{T}$ to $\mathrm{Cs}_{n}^{\mathrm{M}}$ (the set algebra based on $\mathrm{M}[10$, 4.3.4]) via $\phi_{T} \mapsto \phi^{\mathrm{M}}$, where the latter is the set of $n$-ary assignments in $\mathrm{M}$ satisfying $\phi$, omits $\mathbf{X}$. Injectivity follows from the facts that $f$ is non-zero and $\mathfrak{A}$ is simple. For the second part of (1), we use the construction in [23, Thgeorem 4.5], where an atomic $\mathfrak{B} \in \mathrm{Nr}_{n} \mathrm{CA}_{\omega}$ with uncountably many atoms that is not completely representable is constructed. This implies that the maximality condition cannot be dispensed with; else the set of coatoms of $\mathfrak{B}$ call it $X$ will be a non-principal type that cannot be omitted, because any $\mathrm{Gs}_{n}$ omitting $X$ yields a complete representation of $\mathfrak{B}$, witness the last paragraph in [19].

For (2), we can assume that $\mathfrak{A} \subseteq \subseteq_{c} \mathrm{Nr}_{n} \mathfrak{B}, \mathfrak{B} \in \mathrm{Lf}_{\omega}$. We work in $\mathfrak{B}$. Using the notation on [19, p. 216 of proof of Theorem 3.3.4] replacing $\mathfrak{F m}_{T}$ by $\mathfrak{B}$, we have $\mathbf{H}=\bigcup_{i \in \lambda} \bigcup_{\tau \in V} \mathbf{H}_{i, \tau}$ where $\lambda<\mathfrak{p}$, and $V$ is the weak space $\omega_{\omega} \omega^{(I d)}$, can be written as a countable union of nowhere dense sets, and so can the countable union $\mathbf{G}=\bigcup_{j \in \omega} \bigcup_{x \in \mathfrak{B}} \mathbf{G}_{j, x}$. So for any $a \neq 0$, there is an ultrafilter $F \in N_{a} \cap(S \backslash \mathbf{H} \cup \mathbf{G})$ by the Baire category theorem. This induces a homomorphism $f_{a}: \mathfrak{A} \rightarrow \mathfrak{C}_{a}, \mathfrak{C}_{a} \in \mathrm{Cs}_{n}$ that omits the given types, such that $f_{a}(a) \neq 0$. (First one defines $f$ with domain $\mathfrak{B}$ as on p. 216, then restricts $f$ to $\mathfrak{A}$ obtaining $f_{a}$ the obvious way.) The map $g: \mathfrak{A} \rightarrow \mathbf{P}_{a \in \mathfrak{A} \backslash\{0\}} \mathfrak{C}_{a}$ defined via $x \mapsto\left(g_{a}(x): a \in \mathfrak{A} \backslash\{0\}\right)(x \in \mathfrak{A})$ is as required. In case $\mathfrak{A}$ is simple, then by properties of covK, $S \backslash(\mathbf{H} \cup \mathbf{G})$ is non-empty, so if $F \in S \backslash(\mathbf{H} \cup \mathbf{G})$, then $F$ induces a non-zero homomorphism $f$ with domain $\mathfrak{A}$ into a $\boldsymbol{C s}_{n}$ omitting the given types. By simplicity of $\mathfrak{A}$, $f$ is injective. 
Corollary 4.3 .

(1) If $T$ is a countable theory that admits elmination of quantifiers, and $\lambda$ is a cardinal $\left\langle 2^{\aleph_{0}}\right.$, and $\mathbf{F}=\left\langle\Gamma_{i}: i<\lambda\right\rangle$ is a family of complete non-principal types, then $\mathbf{F}$ can be omitted in a countable model of $T$.

(2) If $T$ is any countable theory, then $<\mathfrak{p}$ non-principal types can be omitted; if $T$ is complete, we can further replace $\mathfrak{p}$ by covK.

Proof: Let $T$ be as given in a signature $L$ having $n$ variables. Let $\mathfrak{A}=\mathfrak{F m}_{T}$, and $\mathbf{G}_{i}=\left\{\phi_{T}: \phi \in \Gamma_{i}\right\}$. Then $\mathbf{G}_{i}$ is a a non-principal ultrafilter; maximality follows fom the completeness of types considered. By completeness of $T, \mathfrak{A}$ is simple. Since $T$ admits elimination of quantifiers, then $\mathfrak{F m}_{T} \in \mathrm{Nr}_{n} \mathrm{CA}_{\omega}$. Indeed, let $T_{\omega}$ be the theory in the same signature $L$ but using $\omega$ many variables. Let $\mathfrak{C}=\mathfrak{F m}_{T_{\omega}}$ be the Tarski-Lindenbaum quotient algebra. Then $\mathfrak{C} \in \mathrm{CA}_{\omega}$; in fact $\mathfrak{C} \in \mathbf{I} \mathrm{Cs}_{\omega}$, and the map $\Phi$ defined from $\mathfrak{A}$ to $\mathfrak{N r}_{n} \mathfrak{C}$ via $\phi / \equiv_{T} \mapsto \phi / \equiv_{T_{\omega}}$ is injective and bijective, that is to say, $\Phi$ having domain $\mathfrak{A}$ and codomain $\mathfrak{N r}_{n} \mathfrak{C}$ is in fact onto $\mathfrak{N r}_{n} \mathfrak{C}$ due to quantifier elimination. An application of Theorem 4.2 finishes the proof. The second part is proved exactly like the proof of [19, Theorem 3.2.4] replacing covK by $\mathfrak{p}$.

Here we adress omitting types theorems for certain infinitary extensions of first order logic. Our treatment remains to be purely algebraic. For $\alpha \geq \omega$, we let $\mathrm{Dc}_{\alpha}$ denote the class of dimension complemented $\mathrm{CA}_{\alpha} s$, so that $\mathfrak{A} \in \mathrm{Dc}_{\alpha} \Longleftrightarrow \alpha \backslash \Delta x$ is infinite for every $x \in \mathfrak{A}$.

THEOREM 4.4. Let $\alpha$ be a countable infinite ordinal.

(1) There exists a countable atomic $\mathfrak{A} \in \mathrm{RCA}_{\alpha}$ such that the non-principal types of co-atoms cannot be omitted in a $\mathrm{Gs}_{\alpha}$,

(2) If $\mathfrak{A} \in \mathbf{S}_{c} \mathrm{Nr}_{\alpha} \mathrm{CA}_{\alpha+\omega}$ is countable, $\lambda$ a cardinal $<\mathfrak{p}$ and $\mathbf{X}=\left(X_{i}\right.$ : $i<\lambda)$ is a family of non-principal types, then $\mathbf{X}$ can be omited in a $\mathrm{Gws}_{\alpha}$ (in the sense of definition 2.1 upon replacing $\mathrm{Gs}_{\alpha}$ by $\mathrm{Gws}_{\alpha}$ ).

(3) Assume that the assumption (*) formulated before Theorem 3.20 holds. Then there exists an atomic $\mathfrak{A} \in \mathrm{RCA}_{\alpha}$ such that its DedekindMacNeille completion, namely, $\mathfrak{C} \mathrm{mAt} \mathfrak{A}$ is not in $\mathbf{S N r}_{\alpha} \mathrm{CA}_{\alpha+k}$ for any 
$k \geq 3$. Furthermore, $\mathfrak{A}$ cannot be completely represented by any algebra in $\mathrm{Gws}_{\alpha}$.

\section{ProOF:}

(1) Using exactly the same argument in [11], one shows that if $\mathfrak{C} \in \mathrm{CA}_{\omega}$ is completely representable $\mathfrak{C}=\mathrm{d}_{01}<1$, then $\mid$ At $\mathfrak{C} \mid \geq 2^{\omega}$. The argument is as follows: Suppose that $\mathfrak{C}=\mathrm{d}_{01}<1$. Then there is $s \in h\left(-\mathrm{d}_{01}\right)$ so that if $x=s_{0}$ and $y=s_{1}$, we have $x \neq y$. Fix such $x$ and $y$. For any $J \subseteq \omega$ such that $0 \in J$, set $a_{J}$ to be the sequence with $i$ th co-ordinate is $x$ if $i \in J$, and is $y$ if $i \in \omega \backslash J$. By complete representability every $a_{J}$ is in $h\left(1^{\mathfrak{C}}\right)$ and so it is in $h(x)$ for some unique atom $x$, since the representation is an atomic one. Let $J, J^{\prime} \subseteq \omega$ be distinct sets containing 0 . Then there exists $i<\omega$ such that $i \in J$ and $i \notin J^{\prime}$. So $a_{J} \in h\left(\mathrm{~d}_{0 i}\right)$ and $a_{J}^{\prime} \in h\left(-\mathrm{d}_{0 i}\right)$, hence atoms corresponding to different $a_{J}$ 's with $0 \in J$ are distinct. It now follows that $\mid$ Atc $|=|\{J \subseteq \omega: 0 \in J\} \mid \geq 2^{\omega}$.

Take $\mathfrak{D} \in \mathrm{Cs}_{\omega}$ with universe $\wp\left(\omega_{2}\right)$. Then $\mathfrak{D} \models \mathrm{d}_{01}<1$ and plainly $\mathfrak{D}$ is completely representable. Using the downward Löwenheim-SkolemTarski theorem, take a countable elementary subalgebra $\mathfrak{B}$ of $\mathfrak{D}$. This is possible because the signature of $C A_{\omega}$ is countable. Then in $\mathfrak{B}$ we have $\mathfrak{B}=\mathrm{d}_{01}<1$ because $\mathfrak{B} \equiv \mathfrak{C}$. But $\mathfrak{B}$ cannot be completely representable, because if it were then by the above argument, we get that $|A t \mathfrak{B}| \geq 2^{\omega}$, which is impossible because $\mathfrak{B}$ is countable.

(2) Now we prove the second item, which is a generalization of [19, Theorem 3.2.4]. Though the generalization is strict, in the sense that $\mathrm{Dc}_{\omega} \subsetneq$ $\mathbf{S}_{\mathrm{c}} \mathrm{Nr}_{\omega} \mathrm{CA}_{\omega+\omega}{ }^{1}$ the proof is the same. Without loss, we can take $\alpha=\omega$. Let $\mathfrak{A} \in \mathrm{CA}_{\omega}$ be as in the hypothesis. For brevity, let $\beta=\omega+\omega$. By hypothesis, we have $\mathfrak{A} \subseteq \subseteq_{c} \operatorname{Nr}_{\alpha} \mathfrak{D}$, with $\mathfrak{D} \in \mathrm{CA}_{\beta}$. We can also assume that $\mathfrak{D} \in \mathrm{Dc}_{\beta}$ by replacing, if necessary, $\mathfrak{D}$ by $\mathfrak{S} g^{\mathfrak{D}} \mathfrak{A}$. Since $\mathfrak{A}$ is a complete sublgebra of $\mathrm{Nr}_{\omega} \mathfrak{D}$ which in turn is a complete subalgebra of $\mathfrak{D}$, we have $\mathfrak{A} \subseteq \subseteq_{c} \mathfrak{D}$. Thus given $<\mathfrak{p}$ non-principal types in $\mathfrak{A}$ they stay non-principal in $\mathfrak{D}$. Next one proceeds like in op. cit since $\mathfrak{D} \in \mathrm{Dc}_{\beta}$ is countable; this way omitting any $\mathbf{X}$ consisting of $\langle\mathfrak{p}$ non-principal types. For all non-zero $a \in \mathfrak{D}$, there exists $\mathfrak{B} \in \mathrm{Ws}_{\beta}$ and a homomorphism $f_{a}: \mathfrak{D} \rightarrow \mathfrak{B}$ (not necessarily injective) such that $f_{a}(a) \neq \emptyset$ and $f_{a}$ omits $\mathbf{X}$. Let $\mathfrak{C}=\mathbf{P}_{a \in \mathfrak{D}, a \neq 0} \mathfrak{B}_{a} \in \mathrm{Gws}_{\beta}$. Define

\footnotetext{
${ }^{1}$ It is not hard to see that the full set algebra with universe $\wp\left({ }^{\omega} \omega\right)$ is in $\operatorname{Nr}_{\omega} C A_{\omega+\omega} \subseteq$ $\mathbf{S}_{c} \mathrm{Nr}_{\omega} \mathrm{CA}_{\omega+\omega}$ but it is not in $\mathrm{Dc}_{\omega}$ because for any $s \in{ }^{\omega} U, \Delta\{s\}=\omega$.
} 
$g: \mathfrak{D} \rightarrow \mathfrak{C}$ by $g(x)=\left(f_{a}(x): a \in \mathfrak{D} \backslash\{0\}\right)$, and then relativize $g$ to $\mathfrak{A}$ as follows: Let $W$ be the top element of $\mathfrak{C}$. Then $W=\bigcup_{i \in I}{ }^{\beta} U_{i}^{\left(p_{i}\right)}$, where $p_{i} \in{ }^{\beta} U_{i}$ and ${ }^{\beta} U_{i}^{\left(p_{i}\right)} \cap{ }^{\beta} U_{j}^{\left(p_{j}\right)}=\emptyset$, for $i \neq j \in I$. Let $V=\bigcup_{i \in I}{ }^{\alpha} U_{i}^{\left(p_{i}\lceil\alpha)\right.}$. For $s \in V, s \in{ }^{\alpha} U_{i}^{\left(p_{i}\lceil\alpha)\right.}$ (for a unique $i$ ), let $s^{+}=s \cup p_{i}\lceil\beta \backslash \alpha$. Now define $f: \mathfrak{A} \rightarrow \wp(V)$, via $a \mapsto\left\{s \in V: s^{+} \in g(a)\right\}$. Then $f$ is as required.

The proof of (3) is like the proof of Theorem 3.20

\subsection{Other variants of $L_{\omega, \omega}$}

Now we prove an omitting types theorem for a countable version of the socalled $\omega$-dimensional cylindric polyadic algebras with equality, in symbols $\mathrm{CPE}_{\omega}$, as defined in [8]. Consider the semigroup $\mathrm{T}$ generated by the set of transformations $\{[i \mid j],[i, j], i, j \in \omega$, suc, pred $\}$ defined on $\omega$. Then $\mathrm{T}$ is a strongly rich subsemigroup of $(\omega \omega, \circ)$ in the sense of [18], where suc and pred are the successor and predecessor functions on $\omega$, respectively. For a set $X$, let $\mathfrak{B}(X)$ denote the Boolean set algebra $\langle\wp(X), \cup, \cap, \sim\rangle$. Let $\mathrm{K}_{\mathrm{T}}$ be the class of set algebras of the form $\left\langle\mathfrak{B}(V), \mathrm{C}_{i}, \mathrm{~S}_{\tau}\right\rangle_{i \in \omega, \tau \in \mathrm{T}}$, where $V \subseteq{ }^{\omega} U, V$ is a compressed space, that is $V=\bigcup_{i \in I}{ }^{\alpha} U_{i}^{(p)}$ where for each $i, j \in I, U_{i}=U_{j}$ or $U_{i} \cap U_{j}=\emptyset$. Let $\Sigma_{1}$ be the set of equations defined in [18] axiomatizing $\mathrm{K}_{\mathrm{T}}$; that is $\operatorname{Mod} \Sigma_{1}=\mathrm{K}_{\mathrm{T}}$. Here we do not have diagonal elements in the signature; the corresponding logic is a conservative extension of $L_{\omega, \omega}$ without equality, and it is a proper extension.

Let $\mathrm{Gp}_{\mathrm{T}}$ be the class of set algebras of the form $\left\langle\mathfrak{B}(V), \mathrm{C}_{i}, \mathrm{D}_{i j}, \mathrm{~S}_{\tau}\right\rangle_{i, j \in \omega, \tau \in \mathrm{T}}$, where $V \subseteq{ }^{\omega} U, V$ a non-empty union (not necessarily a disjoint one) of cartesian spaces. Here we have diagonal elements in the signature; the corresponding logic is a variant of $L_{\omega, \omega}$ where quantifiers do not necessarily commute, so $L_{\omega, \omega}$ does not 'embed' in this logic its (square Tarskian) semantics are different. Let $\Sigma_{2}$ be the set of equations defining $\mathrm{CPE}_{\omega}$ in $[8$, Definition 6.3.7] restricted to the countable signature of $\mathrm{Gp}_{\mathrm{T}}$. In the next theorem complete additivity is given explicitly in the second item only. Any algebra $\mathfrak{A}$ satisifying $\Sigma_{2}$ is completely additive (due to the presence of diagonal elements), cf. [8].

THEOREM 4.5 .

(1) If $\mathfrak{A}=\Sigma_{2}$ is countable and $\mathbf{X}=\left(X_{i}: i<\lambda\right), \lambda<\mathfrak{p}$ is a family of subsets of $\mathfrak{A}$, such that $\prod X_{i}=0$ for all $i<\lambda$, then there exists 
$\mathfrak{B} \in \mathrm{Gp}_{\mathrm{T}}$ and an isomorphism $f: \mathfrak{A} \rightarrow \mathfrak{B}$ such that $\bigcap_{x \in X_{i}} f(x)=\emptyset$ for all $i<\lambda$.

(2) If $\mathfrak{A}=\Sigma_{1}$ is countable, and completely additive and $\mathbf{X}=\left(X_{i}: i<\lambda\right)$, $\lambda<\mathfrak{p}$ is a family of subsets of $\mathfrak{A}$, such that $\prod X_{i}=0$ for all $i<\lambda$, then there exists $\mathfrak{B} \in \mathrm{K}_{\mathrm{T}}$ and an isomorphism $f: \mathfrak{A} \rightarrow \mathfrak{B}$ such that $\bigcap_{x \in X_{i}} f(x)=\emptyset$ for all $i<\lambda$.

(3) In particular, for both cases any countable atomic algebra is completely representable.

Proof: For brevity, throughout the proof of the first two items, let $\alpha=$ $\omega+\omega$. By strong richness of $\mathrm{T}$, it can be proved that $\mathfrak{A}=\mathfrak{N r}_{\omega} \mathfrak{B}$ where $\mathfrak{B}$ is an $\alpha$-dimensional dilation with substitution operators coming from a countable subsemigroup $S \subseteq\left({ }^{\alpha} \alpha, \circ\right)$ [22]. It suffices to show that for any non-zero $a \in \mathfrak{A}$, there exist a countable $\mathfrak{D} \in \mathrm{Gp}_{\mathrm{T}}$ and a homomorphism (that is not necessarily injective) $f: \mathfrak{A} \rightarrow \mathfrak{D}$, such that $\bigcap_{x \in X_{i}} f(x)=\emptyset$ for all $i \in \omega$ and $f(a) \neq 0$. So fix non-zero $a \in \mathfrak{A}$. For $\tau \in \mathrm{S}$, set $\operatorname{dom}(\tau)=\{i \in \alpha: \tau(i) \neq i\}$ and $\operatorname{rng}(\tau)=\{\tau(i): i \in \operatorname{dom}(\tau)\}$. Let adm be the set of admissible substitutions in S, where now $\tau \in \operatorname{adm}$ if $\operatorname{dom} \tau \subseteq \omega$ and $\operatorname{rng} \tau \cap \omega=\emptyset$. Since $S$ is countable, we have $|\mathrm{adm}| \leq \omega$; in fact it can be easily shown that $|\mathrm{adm}|=\omega$. Then for all $i<\alpha, p \in \mathfrak{B}$ and $\sigma \in \operatorname{adm}, \mathrm{s}_{\sigma} \mathrm{c}_{i} p=$ $\sum_{j \in \alpha} \mathrm{s}_{\sigma} \mathrm{s}_{j}^{i} p$. By $\mathfrak{A}=\mathfrak{N r}_{\omega} \mathfrak{B}$ we also have, for each $i<\omega, \prod^{\mathfrak{B}} X_{i}=0$, since $\mathfrak{A}$ is a complete subalgebra of $\mathfrak{B}$. Because substitutions are completely additive, for all $\tau \in$ adm and all $i<\lambda, \prod \mathrm{s}_{\tau}^{\mathfrak{B}} X_{i}=0$. For better readability, for each $\tau \in$ adm, for each $i \in \omega$, let $X_{i, \tau}=\left\{\mathrm{s}_{\tau} x: x \in X_{i}\right\}$. Then by complete additivity, we have: $(\forall \tau \in$ adm $)(\forall i \in \lambda) \prod^{\mathfrak{B}} X_{i, \tau}=0$. Let $S$ be the Stone space of $\mathfrak{B}$, whose underlying set consists of all Boolean ultrafilters of $\mathfrak{B}$ and for $b \in B$, let $N_{b}$ denote the clopen set consisting of all ultrafilters containing $b$. Then from the suprema obtained above, it follows that for $x \in \mathfrak{B}, j<\alpha, i<\lambda$ and $\tau \in$ adm, the sets $\mathbf{G}_{\tau, j, x}=$ $N_{\mathbf{s}_{\tau} \mathrm{c}_{j} x} \backslash \bigcup_{i} N_{\mathbf{s}_{\tau} \mathbf{s}_{i}^{j} x}$ and $\mathbf{H}_{i, \tau}=\bigcap_{x \in X_{i}} N_{\mathbf{s}_{\tau} x}$ are closed nowhere dense sets in $S$. Also each $\mathbf{H}_{i, \tau}$ is closed and nowhere dense. Like before, we can assume that $\mathfrak{B}$ is countable by assuming that $\mathfrak{A}$ generates $\mathfrak{B}$ is the presence of $\mid$ alpha $\mid=(|A|=\omega)$ many operations. Let $\mathbf{G}=\bigcup_{\tau \in \operatorname{adm}} \bigcup_{i \in \alpha} \bigcup_{x \in B} \mathbf{G}_{\tau, i, x}$ and $\mathbf{H}=\bigcup_{i \in \lambda} \bigcup_{\tau \in \text { adm }} \mathbf{H}_{i, \tau}$. Then $\mathbf{H}$ is meager, that is it can be written as a countable union of nowghere dense sets. This follows from the properties of $\mathfrak{p}$ By the Baire Category theorem for compact Hausdorff spaces, we get that $X=S \backslash \mathbf{H} \cup \mathbf{G}$ is dense in $S$, since $\mathbf{H} \cup \mathbf{G}$ is meager, because 
$\mathbf{G}$ is meager, too, since adm, $\alpha$ and $\mathfrak{B}$ are all countable. Accordingly, let $F$ be an ultrafilter in $N_{a} \cap X$, then by its construction $F$ is a perfect ultrafilter [20, p. 128]. Let $\Gamma=\left\{i \in \alpha: \exists j \in \omega: c_{i} \mathrm{~d}_{i j} \in F\right\}$. Since $\mathrm{c}_{i} \mathrm{~d}_{i i}=1$, then $\omega \subseteq \Gamma$. Furthermore the inclusion is proper, because for every $i \in \omega$, there is a $j \in \alpha \backslash \omega$ such that $\mathrm{d}_{i j} \in F$. Define the relation $\sim$ on $\Gamma$ via $m \sim n \Longleftrightarrow \mathrm{d}_{m n} \in F$. Then $\sim$ is an equivalence relation because for all $i, j, k \in \alpha, \mathrm{d}_{i i}=1 \in F, \mathrm{~d}_{i j}=\mathrm{d}_{j i}, \mathrm{~d}_{i k} \cdot \mathrm{d}_{k j} \leq \mathrm{d}_{l k}$ and filters are closed upwards. Now we show that the required representation will be a $\mathrm{Gp}_{\mathrm{T}}$ with base $M=\Gamma / \sim$. One defines the homomorphism $f$ using the hitherto obtained perfect ultrafilter $F$ as follows: For $\tau \in{ }^{\omega} \Gamma$, such that $\operatorname{rng}(\tau) \subseteq \Gamma \backslash \omega$ (the last set is non-empty, because $\omega \subsetneq \Gamma$ ), let $\bar{\tau}: \omega \rightarrow M$ be defined by $\bar{\tau}(i)=\tau(i) / \sim$ and write $\tau^{+}$for $\tau \cup I d_{\alpha \backslash \omega}$. Then $\tau^{+} \in$ adm, because $\tau^{+} \uparrow \omega=\tau, \operatorname{rng}(\tau) \cap \omega=\emptyset$, and $\tau^{+}(i)=i$ for all $i \in \alpha \backslash \omega$. Let $V=\left\{\bar{\tau} \in{ }^{\omega} M: \tau: \omega \rightarrow \Gamma, \operatorname{rng}(\tau) \cap \omega=\emptyset\right\}$. Then $V \subseteq{ }^{\omega} M$ is non-empty (because $\omega \subsetneq \Gamma$ ). Now define $f$ with domain $\mathfrak{A}$ via: $a \mapsto\left\{\bar{\tau} \in V: \mathrm{s}_{\tau^{+}}^{\mathfrak{B}} a \in F\right\}$. Then $f$ is well defined, that is, whenever $\sigma, \tau \in{ }^{\omega} \Gamma$ and $\tau(i) \backslash \sigma(i)$ for all $i \in \omega$, then for any $x \in \mathfrak{A}, \mathrm{s}_{\tau^{+}}^{\mathfrak{B}} x \in F \Longleftrightarrow \mathrm{s}_{\sigma^{+}}^{\mathfrak{B}} x \in F$. Furthermore $f(a) \neq 0$, since $\mathrm{s}_{I d} a=a \in F$ and $I d$ is clearly admissable. The congruence relation just defined on $\Gamma$ guarantees that the hitherto defined homomorphism respects the diagonal elements. As before, for the other operations, preservation of cylindrifiers is guaranteed by the condition that $F \notin G_{\tau, i, p}$ for all $\tau \in$ adm, $i \in \alpha$ and all $p \in A$. For omitting the given family of non-principal types, we use that $F$ is outside $\mathbf{H}$, too. This means (by definition) that for each $i<\lambda$ and each $\tau \in$ adm there exists $x \in X_{i}$, such that $\mathrm{s}_{\tau}^{\mathfrak{B}} x \notin F$. Let $i<\lambda$. If $\bar{\tau} \in V \cap \bigcap_{x \in X_{i}} f(x)$, then $\mathrm{s}_{\tau^{+}}^{\mathfrak{B}} x \in F$ which is impossible because $\tau^{+} \in \operatorname{adm}$. We have shown that for each $i<\omega$, $\bigcap_{x \in X_{i}} f(x)=\emptyset$.

For the second required one deals with all substitutions in the semigroup $\mathrm{S}$ determining the signature of the dilation not just adm, namely, the admissable ones as defined above. More succintly, now all substitutions in S are admissable. Other than that, the idea is essentially the same appealing to the Baire category theorem. Let $\mathrm{T}$ be as above. Assume that $\mathfrak{A} \models \Sigma_{1}$ is countable, and fix non-zero $a \in \mathfrak{A}$. Similarly to the first part we will construct a set algebra $\mathfrak{C}$ in $\mathrm{K}_{\mathrm{T}}$ and a homomorphism $f: \mathfrak{A} \rightarrow \mathfrak{C}$ omitting the given non-principal types and satisfying that $f(a) \neq 0$. By [18], there exists $\mathfrak{B}$ such that $\mathfrak{A}=\operatorname{Nr}_{\omega} \mathfrak{B}$ and the signature of $\mathfrak{B}$ has, besides all the Boolean operations, all cylindrifiers $\mathrm{c}_{i}: i \in \alpha$, and the substitutions are determined by a semigroup defined from the rich semigroup T. Substitu- 
tions in the signature of $\mathfrak{B}$ are indexed by transformations in $\mathrm{S}$; which we explicitly describe. The semigroup $\mathrm{S}$ is the subsemigroup of ${ }^{\alpha} \alpha$ generated by the set $\{\bar{\tau}: \tau \in \mathrm{T}\}$ together with all replacements and transpositions on $\alpha$. Here $\bar{\tau}$ is the transformation that agrees with $\tau$ on $\omega$ and otherwise is the identity. For all $i<\alpha, p \in \mathfrak{B}$, we have $\mathrm{c}_{i} p=\sum_{j \in \alpha} \mathrm{s}_{j}^{i} p$.

By $\mathfrak{A}=\mathrm{Nr}_{\omega} \mathfrak{B}$ we also have, for each $i<\omega, \prod^{\mathfrak{B}} X_{i}=0$, since $\mathfrak{A}$ is a complete subalgebra of $\mathfrak{B}$. Let $V$ be the generalized $\omega$-dimensional weak space $\bigcup_{\tau \in S}{ }^{\omega} \alpha^{(\tau)}$. Recall that ${ }^{\omega} \alpha^{(\tau)}=\left\{s \in{ }^{\omega} \alpha:\left|\left\{i \in \omega: s_{i} \neq \tau_{i}\right\}\right|<\omega\right\}$. For each $\tau \in V$ and for each $i \in \lambda$, let $X_{i, \tau}=\left\{\mathrm{s}_{\bar{\tau}}^{\mathfrak{B}} x: x \in X_{i}\right\}$. Here we are using that for any $\tau \in V, \bar{\tau} \in \mathrm{S}$. By complete additivity which is given as an assumption, it follows that $(\forall \tau \in V)(\forall i \in \kappa) \prod^{\mathfrak{B}} X_{i, \tau}=0$.

Let $S$ denote the Stone space of the boolean part of $\mathfrak{B}$. Like before, for $p \in B$, let $N_{p}$ be the clopen set of $S$ consisting of all ultrafilters of the boolean part of $\mathfrak{B}$ containing $p$. Then for $x \in \mathfrak{B}, j<\alpha, i<\lambda, \tau \in \mathrm{S}$ (using the suprema just established), the sets $\mathbf{G}_{j, x}=N_{\mathrm{c}_{j} x} \backslash \bigcup_{i} N_{\mathrm{s}_{i}^{j} x}$ and $\mathbf{H}_{i, \tau}=$ $\bigcap_{x \in X_{i}} N_{\mathrm{s}_{\tau} x}$ are closed nowhere dense sets in $S$. Also each $\mathbf{H}_{i, \tau}$ is closed and nowhere dense.

Let $\mathbf{G}=\bigcup_{i \in \alpha} \bigcup_{x \in B} \mathbf{G}_{i, x}$ and $\mathbf{H}=\bigcup_{i \in \lambda} \bigcup_{\tau \in S} \mathbf{H}_{i, \tau}$. Then $\mathbf{H}$ is meager, since it is a countable union of nowhere dense sets. Once more by the Baire Category theorem for compact Hausdorff spaces, we get that $X=$ $S \backslash \mathbf{H} \cup \mathbf{G}$ is dense in $S$, Let $F$ be an ultrafilter in $N_{a} \cap X$. One builds the required represention from $F$ as follows [18]: Let $\wp(V)$ be the full boolean set algebra with unit $V$. Let $f$ be the function with domain $A$ such that $f(a)=\left\{\tau \in V: \mathbf{s}_{\bar{\tau}}^{B} a \in F\right\}$. Then $f$ is the desired homorphism from $\mathfrak{A}$ into the set algebra $\left\langle\wp(V), \mathbf{c}_{i}, \mathbf{s}_{\tau}\right\rangle_{i \in \omega, \tau \in \mathrm{T}}$. In particular, $f(a) \neq 0$, because $I d \in f(a)$. That $f$ omits the given non-principal types is exactly like the first part, modulo replacing adm by (the whole of the semigroup) $\mathrm{S}$.

Given $\mathfrak{A}$ as in the hypothesis, the last required follows by omitting the non-principal type consisting of co-atoms obtaining a complete representation of $\mathfrak{A}$.

The cylindric reduct of the algebra $\mathfrak{T m A t}$ in the proof of Theorem 3.5 is representable, but not completely representable, for a complete representation of $\mathfrak{T} \mathfrak{m A t}$ induces an ordinary representation for $\mathfrak{C m A t}$. In fact, it is known that for $2<n<\omega$ the class CRCA $_{n}$ is not elementary [11]. We give a short proof. Let $\mathfrak{A} \in \mathrm{Nr}_{n} \mathrm{CA}_{\omega}$ be an atomic algebra with uncountable many atoms having no complete representation. This algebra exists 
[23, Theorem 4.5]. Let $\mathrm{LCA}_{n}$ be the class of $\mathrm{CA}_{n} \mathrm{~s}$ satifying the Lyndon conditions in the sense of [13]. Then using Lemma 3.3, $\exists$ has a winning strategyin $\mathbf{G}^{\omega}($ At $\mathfrak{A})$, hence she has winning strategyin $G_{\omega}^{\omega}($ At $\mathfrak{A})$, a fortiori in the usual $k$ rounded atomic game $G_{k}($ At $\mathfrak{A})$ for all $k \in \omega$. Thus by definition $\mathfrak{A} \in \mathrm{LCA}_{n}$. But LCA $\mathrm{LA}_{n}$ is the elementary closure of $\mathrm{CRCA}_{n}$ and we are done. For a class $\mathrm{K}$, let $\mathrm{K}^{\text {ad }}$ be the class of completey additive algebras in K. In contrast for polyadic (equality) algebras of infinite dimension, we have the following result proved in $[21,23]$. We give a unifted proof.

THEOREM 4.6. Let $\alpha$ be an infinite ordinal and $n<\omega(\leq \alpha)$. If $\mathfrak{D} \in \mathrm{PEA}_{\alpha}$ $\left(\mathrm{PA}_{\alpha}\right.$ is completely additive and) is atomic, then any complete subalgebra of $\mathfrak{N} r_{n} \mathfrak{D}$ is completely representable as a $\mathrm{PEA}_{n}\left(\mathrm{PA}_{n}\right)$. In particular, $\mathbf{S}_{c} \mathrm{PA}_{\alpha}^{\mathrm{ad}} \cap \mathbf{A t}=\mathrm{PA}_{\alpha}^{\text {ad }} \cap \mathbf{A t}=\mathrm{CRPA}_{\alpha}$ and the class $\mathrm{CRPA}_{\alpha}$ is elementary.

Proof sketch. Assume that $\mathfrak{A} \subseteq_{c} \mathfrak{N r}_{n} \mathfrak{D}$, where $\mathfrak{D} \in \mathrm{PEA}_{\alpha}$ is atomic. Let $c \in \mathfrak{A}$ be non-zero. We will find a homomorphism $f: \mathfrak{A} \rightarrow \wp\left({ }^{n} U\right)$ such that $f(c) \neq 0$, and preserves infinitary joins. Assume for the moment (to be proved in a while) that $\mathfrak{A} \subseteq$. Then by [12, Lemma 2.16] $\mathfrak{A}$ is atomic because $\mathfrak{D}$ is. For brevity, let $X=$ At $\mathfrak{A}$. Let $\mathfrak{m}$ be the local degree of $\mathfrak{D}, \mathfrak{c}$ its effective cardinality and let $\beta$ be any cardinal such that $\beta \geq \mathfrak{c}$ and $\sum_{s<\mathfrak{m}} \beta^{s}=\beta$; such notions are defined in [6]. We can assume that $\mathfrak{D}=\mathfrak{N \mathfrak { r }}_{\alpha} \mathfrak{B}$, with $\mathfrak{B} \in \operatorname{PEA}_{\beta}[10$, Theorem 5.4.17]. For any ordinal $\mu \in \beta$, and $\tau \in{ }^{\mu} \beta$, write $\tau^{+}$for $\tau \cup I d_{\beta \backslash \mu}(\in \beta \beta)$. Consider the following family of joins evaluated in $\mathfrak{B}$, where $p \in \mathfrak{D}, \Gamma \subseteq \beta$ and $\tau \in{ }^{\alpha} \beta$ : $\left(^{*}\right.$ ) $\mathrm{c}_{(\Gamma)} p=\sum^{\mathfrak{B}}\left\{\mathbf{s}_{\tau^{+}} p: \tau \in{ }^{\omega} \beta, \quad \tau \mid \alpha \backslash \Gamma=I d\right\}$, and $\left({ }^{* *}\right): \sum \mathrm{s}_{\tau^{+}}^{\mathfrak{B}} X=1$. The first family of joins exists [6, Proof of Theorem 6.1], and the second exists, because $\sum^{\mathfrak{A}} X=\sum^{\mathfrak{D}} X=\sum^{\mathfrak{B}} X=1$ and $\tau^{+}$is completely additive, since $\mathfrak{B} \in \mathrm{PEA}_{\beta}$. The last equality of suprema follows from the fact that $\mathfrak{D}=\mathfrak{N r}_{\alpha} \mathfrak{B} \subseteq_{c} \mathfrak{B}$ and the first from the fact that $\mathfrak{A} \subseteq \subseteq_{c} \mathfrak{D}$. All this is proved in [23]. Let $F$ be any Boolean ultrafilter of $B$ generated by an atom below $a$. We show that $F$ will preserve the family of joins in $(*)$ and $(* *)$. While in proving a positive a OTT for $L_{n}$ in item (2) of Theorem 4.2 we resorted to the Baire Category Theorem, now we use a far more basic less sophisticated topological argument. One forms nowhere dense sets in the Stone space of $\mathfrak{B}$ corresponding to the aforementioned family of joins as follows: The Stone space of (the Boolean reduct of) $\mathfrak{B}$ has underlying set $S$, the set of all Boolean ultrafilters of $\mathfrak{B}$. For $b \in \mathfrak{B}$, let $N_{b}$ be the clopen set $\{F \in S: b \in F\}$. The required nowhere dense sets are defined for $\Gamma \subseteq \beta, p \in \mathfrak{D}$ and $\tau \in{ }^{\alpha} \beta$ via: $A_{\Gamma, p}=N_{\mathrm{c}_{(\Gamma)} p} \backslash \bigcup_{\tau: \alpha \rightarrow \beta} N_{\mathrm{s}_{\tau}+}$, 
and $A_{\tau}=S \backslash \bigcup_{x \in X} N_{\mathrm{s}_{\tau^{+}} x}$. The principal ultrafilters are isolated points in the Stone topology, so they lie outside the nowhere dense sets defined above. Hence any such ultrafilter preserve the joins in $\left(^{*}\right)$ and $\left({ }^{*}\right)$. Fix a principal ultrafilter $F$ with $a \in F$. Define the equivalence relation $E$ (on $\beta$ ) by setting $i E j \Longleftrightarrow \mathrm{d}_{i j}^{\mathfrak{B}} \in F(i, j \in \beta)$. Define $f: \mathfrak{A} \rightarrow \wp\left({ }^{n}(\beta / E)\right)$, via $x \mapsto\left\{\bar{t} \in{ }^{n}(\beta / E): \mathrm{s}_{t \cup I d_{\beta \sim n}}^{\mathfrak{B}} x \in F\right\}$, where $\bar{t}(i / E)=t(i)(i<n)$ and $t \in{ }^{n} \beta$. Then $f$ is a well-defined homomorphism; preserving cylindrifiers depends on $\left(^{*}\right)$. $f$ defines a complete representation such that Also $f(c) \neq 0$ because $I d \in f(c)$. To show that $f$ is an atomic, hence complete representation, one uses (**) as follows: By construction, for every $s \in{ }^{n}(\beta / E)$, there exists $x \in X(=$ Ata $)$, such that $\mathrm{s}_{s \cup I d_{\beta \sim n}}^{\mathfrak{B}} x \in F$, from which we get $\bigcup_{x \in X} f(x)=$ $n(\beta / E)$. If $\mathfrak{A} \in \mathrm{PA}_{\alpha}$, we do not need to bother about diagonal elements and so the base of the representation will be simply $\beta$ (as defined above for $\mathrm{PEA}_{\alpha}$ ), not $\beta / E$, and the desired homomorphism, with $n \leq \alpha$, is defined via $g: \mathfrak{A} \rightarrow \wp\left({ }^{n} \beta\right)$, via $\left.x \mapsto \mathrm{t} \in{ }^{n} \beta: \mathrm{s}_{t \cup I d_{\beta \sim n}}^{\mathfrak{B}} x \in F\right\}$. Checking that $g$ preserves the operations and that $g$ is atomic, hence complete, is exactly like the PEA case. For $\mathrm{PA}_{\alpha}$, atomicity can be expressed by a first order sentence, and complete additivity can be captured by continuum many first order formulas [21]

\section{Concluding remarks and related results}

(1) A Theorem of Vaught in basic model theory, says that a countable atomic $L_{\omega, \omega}$ theory $T$ has a unique atomic (equivalently in this context prime) model. This can be proved by a direct application of the clssical Orey-Henkin Omitting Types Theorem. The unique atomic atomic model is the 'smallest' models of $T$, in the sense that it elementary embeds into other models of $T$. The last theorem says that Keisler's logics which allow formulas of infinite length and quantification on infinitely many variables, enjoys a form of Vaught's theorem. And in Keisler's logics there is the additional advantage that there is no restrictions on the cardinality of atomic theories (algebras) considered. For $L_{\omega, \omega}$, Vaught's theorem is known to fail for theories having uncountable cadinality. If $T$ is an atomic theory in Keisler's logic, and the Tarski-Lindenbaum atomic quotient algebra $\mathfrak{F m}_{T}$ happens to be completely additve, then $T$ has an atomic model. In contrast, in Corollary 3.13, we actually showed that Vaught's theorem fails for $L_{n}$ when we substantially broaden the class of permissable models; it 
fails even for ' $n+3$-square models.' For $2<n<\omega$, there is a countable atomic $L_{n}$ theory that lacks even an atomic $n+3$-square model (let alone an ordinary atomic model), i.e a complete $n+3$-square representation of the Tarski-Lindenbaum quotient algebra $\mathfrak{F m}_{T}\left(\in \mathrm{RCA}_{n}\right)$.

(2) Let $2<n<l \leq m \leq \omega$. Consider the statemet notVT $(l, m)$ : There exists a countable, complete and atomic $L_{n}$ first order theory $T$ in a signature $L$ such that the type $\Gamma$ consisting of co-atoms in the cylindric TarskiLindenbaum quotient algebra $\mathfrak{F m}_{T}$ is realizable in every $m$-square model, but $\Gamma$ cannot be isolated using $\leq l$ variables, where $n \leq l<m \leq \omega$. An $m$-square model of $T$ is an $m$-square represenation of $\mathfrak{F m}_{T}$. The statement $\operatorname{not} \mathrm{VT}(\mathrm{I}, \mathrm{m})$, short for Vaught's Theorem (VT) fails at (the parameters) $l$ and $m$. Let $\mathrm{VT}(l, m)$ stand for $\mathrm{VT}$ holds at $l$ and $m$, so that by definition $\operatorname{not} \mathrm{VT}(l, m) \Longleftrightarrow \neg \mathrm{VT}(l, m)$. We also include $l=\omega$ in the equation by defining $\mathrm{VT}(\omega, \omega)$ as VT holds for $L_{\omega, \omega}$ : Atomic countable first order theories have atomic countable models. For $2<n<l \leq m \leq \omega$ and $l=m=\omega$, it is likely and plausible that $\left({ }^{* * *}\right): \mathrm{VT}(l, m) \Longleftrightarrow l=m=\omega$. In other words: Vaught's theorem holds only in the limiting case when $l \rightarrow \infty$ and $m=\omega$ and not 'before'. We give sufficient condition for $(* * *)$ to happen. The following definition to be used in the sequel is taken from [2]:

Definition 5.1. [2, Definition 3.1] Let $\mathfrak{R}$ be a relation algebra, with nonidentity atoms $I$ and $2<n<\omega$. Assume that $J \subseteq \wp(I)$ and $E \subseteq{ }^{3} \omega$. We say that $(J, E)$ is a strong $n$-blur for $\mathfrak{R}$ if it $(J, E)$ is an $n$-blur of $R$ in the sense of [2, Definition 3.1], that is to say $J$ is a complex $n$ blur and $E$ is an index blur such that the complex $n$-blur satisfies:

$$
\left(\forall V_{1}, \ldots V_{n}, W_{2}, \ldots W_{n} \in J\right)(\forall T \in J)(\forall 2 \leq i \leq n) \operatorname{safe}\left(V_{i}, W_{i}, T\right) .
$$

Theorem 5.2. For $2<n<\omega$ and $n \leq l<\omega$, $\operatorname{notVT}(n, n+3)$ and $\operatorname{not} \mathrm{VT}(l, \omega)$ hold. Furthermore, if for each $n<m<\omega$, there exists a finite relation algebra $\mathfrak{R}_{m}$ having $m-1$ strong blur and no $m$-dimensional relational basis, then (***) above for VT holds.

Proof: We start by the last part. Let $\mathfrak{R}_{m}$ be as in the hypothesis with strong $m-1$-blur $(J, E)$ and $m$-dimensional relational basis. We 'blow up and blur' $\mathfrak{R}_{m}$ in place of the Maddux algebra $\mathfrak{E}_{k}(2,3)$ blown up and blurred in [2, Lemma 5.1], where $k<\omega$ is the number of non-identity atoms and $k$ depends recursively on $l$, giving the desired'strong' $l$-blurness, 
cf. [2, Lemmata 4.2, 4.3]. The relation algebra $\mathfrak{B} b\left(\Re_{m}, J, E\right)$, obtained by blowing up and blurring $\mathfrak{R}_{m}$ with respect to $(J, E)$, is $\mathfrak{T} \mathbf{m A t}$ (the term algebra). For brevity call it $\mathcal{R}$. Now take $\mathfrak{A}=\mathfrak{B b}_{n}\left(\mathfrak{R}_{m}, J, E\right)$ as defined in [2] to be the $\mathrm{CA}_{n}$ obtained after blowing up and blurring $\mathfrak{R}_{m}$ to a weakly representable relation algebra atom structure, namely, $\mathbf{A t}=\mathrm{At} \mathcal{R}$. Here by [2, Theorem 3.29 (iii)], Mat ${ }_{n} \mathrm{At} \mathcal{R}$ (the set of $n$-basic matrices on $A t \mathcal{R})$ is a $C_{n}$ atom structure and $\mathfrak{A}$ is an atomic subalgebra of $\mathfrak{C m M a t}_{n}($ At $\mathcal{R})$ containing $\mathfrak{T m M a t}_{n}($ At $\mathcal{R})$, cf. [2]. In fact, by [2, item (3) p. 80], $\mathfrak{A} \cong \mathfrak{N r}_{n} \mathfrak{B} b_{l}\left(\mathfrak{R}_{m}, J, E\right)$. The last algebra $\mathfrak{B} b_{l}\left(\mathfrak{R}_{m}, J, E\right)$ is defined and the isomorphism holds because $\mathfrak{R}_{m}$ has a strong $l$-blur. The embedding $h: \mathfrak{R} \mathfrak{d}_{n} \mathfrak{B} \mathfrak{b}_{l}\left(\mathfrak{R}_{m}, J, E\right) \rightarrow \mathfrak{A}$ defined via $x \mapsto\{M \uparrow n: M \in x\}$ restricted to $\mathfrak{N r}_{n} \mathfrak{B} b_{l}\left(\mathfrak{R}_{m}, J, E\right)$ is an isomorphism onto $\mathfrak{A}[2$, p. 80]. Surjectiveness uses the displayed condition in Definition 5.1 of strong l-blurness. Then $\mathfrak{A} \in \mathrm{RCA}_{n} \cap \mathrm{Nr}_{n} \mathrm{CA}_{l}$, but $\mathfrak{A}$ has no complete $m$-square representa-

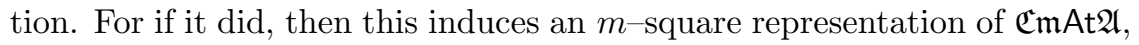
But $\mathfrak{C} \mathfrak{m A t} \mathfrak{A}$ does not have an $m$-square representation, because $\mathfrak{R}$ does not have an $m$-dimensional relational basis, and $\mathfrak{R} \subseteq \mathfrak{R a C m A t} \mathfrak{A}$. So an $m$-square representation of $\mathfrak{C} \mathfrak{m} A$ t $\mathfrak{A}$ induces one of $\mathfrak{R}$ which that $\mathfrak{R}$ has no $m$-dimensional relational basis, a contradiction. We prove notVT $(m-1, m)$, hence the required, namely, $(* * *)$. By $[10, \S 4.3]$, we can (and will) assume that $\mathfrak{A}=\mathfrak{F m}_{T}$ for a countable, simple and atomic theory $L_{n}$ theory $T$. Let $\Gamma$ be the $n$-type consisting of co-atoms of $T$. Then $\Gamma$ is realizable in every $m$-square model, for if $\mathrm{M}$ is an $m$-square model omitting $\Gamma$, then $\mathrm{M}$ would be the base of a complete $m$-square representation of $\mathfrak{A}$, and so by Theorem $3.9 \mathfrak{A} \in \mathbf{S}_{c} \mathrm{Nr}_{n} \mathrm{D}_{m}$ which is impossible. Suppose for contradiction that $\phi$ is an $m-1$ witness, so that $T \models \phi \rightarrow \alpha$, for all $\alpha \in \Gamma$, where recall that $\Gamma$ is the set of coatoms. Then since $\mathfrak{A}$ is simple, we can assume without loss that $\mathfrak{A}$ is a set algebra with base $M$ say. Let $\mathrm{M}=\left(M, R_{i}\right)_{i \in \omega}$ be the corresponding model (in a relational signature) to this set algebra in the sense of $[10, \S 4.3]$. Let $\phi^{\mathrm{M}}$ denote the set of all assignments satisfying $\phi$ in M. We have $\mathrm{M}=T$ and $\phi^{\mathrm{M}} \in \mathfrak{A}$, because $\mathfrak{A} \in \mathrm{Nr}_{n} \mathrm{CA}_{m-1}$. But $T=\exists x \phi$, hence $\phi^{\mathrm{M}} \neq 0$, from which it follows that $\phi^{\mathrm{M}}$ must intersect an atom $\alpha \in \mathfrak{A}$ (recall that the latter is atomic). Let $\psi$ be the formula, such that $\psi^{\mathrm{M}}=\alpha$. Then it cannot be the case that $T \models \phi \rightarrow \neg \psi$, hence $\phi$ is not a witness, contradiction and we are done. Finally, $\operatorname{notVT}(n, n+3)$ and $\operatorname{notVT}(l, \omega)$ $(n \leq l<\omega)$ follow from Theorm 3.5 and [2] using the same reasoning as above. 
(3) Let $2<n<\omega$. For any $m>n$ there exists an $n$-variable formula that cannot be proved using $m-1$ variables, but can be proved using $m$ variables [12, Theorem 15.17], using any standard Hilbert style proof system $[10, \S 4.3]$. To prove this, for each $m>n+1$ Hirsch and Hodkinson constructed a finite relation algebra, such that $\mathfrak{R}_{m}$ has an $m-1$ dimensional hyperbasis, but no $m$-dimensional hyperbasis $[12, \S 15.2-15.4]$. To prove that VT fails everywhere, as defined above, one needs to construct, for each $n+1<m<\omega$, a finite relation algebra $\mathfrak{R}_{m}$ having a strong $m-1$ blur, but no $m$-dimensional basis. In this case blowing up and blurring $\mathfrak{R}_{m}$ gives $\mathrm{a}(\mathrm{n}$ infinite) relation algebra having an $m-1$ dimensional cylindric basis, whose Dedekind-MacNeille completion has no $m$-dimensional basis.

(4) Coming back full circle we reprove strong non-finite axiomatizibility results refining Monk's obtained by Maddux and Biro. Let $2<n \leq l<$ $m \leq \omega$. In $\mathrm{VT}(l, m)$, while the parameter $l$ measures how close we are to $L_{\omega, \omega}, m$ measures the 'degree' of squareness of permitted models. Using elementary calculas terminology one can view $\lim _{l \rightarrow \infty} \mathrm{VT}(l, \omega)=\mathrm{VT}(\omega, \omega)$ algebraically using ultraproducts as follows. Fix $2<n<\omega$. For each $2<$ $n \leq l<\omega$, let $\mathfrak{R}_{l}$ be the finite Maddux algebra $\mathfrak{E}_{f(l)}(2,3)$ with strong $l$-blur $\left(J_{l}, E_{l}\right)$ and $f(l) \geq l$ as specified in [2, Lemma 5.1] (denoted by $k$ therein). Let $\mathcal{R}_{l}=\mathfrak{B b}\left(\Re_{l}, J_{l}, E_{l}\right) \in$ RRA and let $\mathfrak{A}_{l}=\mathfrak{N r}_{n} \mathfrak{B b}_{l}\left(\mathfrak{R}_{l}, J_{l}, E_{l}\right) \in \mathrm{RCA}_{n}$. Then $\left(\right.$ At $\left.\mathcal{R}_{l}: l \in \omega \sim n\right)$, and (At: $\left.\mathfrak{A}_{l}: l \in \omega \sim n\right)$ are sequences of weakly representable atom structures that are not strongly representable with a completely representable ultraproduct.

Corollary 5.3. Let $2<n<\omega$. Then the varieties $\mathrm{RCA}_{n}$ and RRA, together with any finite first order definable expansion of each, cannot be derived from any finite set of equations valid in the variety [3, 17].

We used a rainbow construction to show ultimatey that the $m$-clique guraded-fragments of $L_{n}$ with respect to $m$ square and $m$ flat models, equivalently the $m$-packed fragments of $L_{n}$ are not Sahlqvist. We show thay $\operatorname{not} \mathrm{VT}(l, m)$ fails on the 'horizontal $x$ axis' and the 'vertical $y$-axis.' To show that VTfails everywhere, that is to prove that $\mathrm{VT}(\mathrm{I}, \mathrm{m}) \Longleftrightarrow$ $l=m=\omega$, we reduced the problem in Theorem 5.2 to finding a finite relation algebra having a strong $l$ blur and no $m$-dimensional relational basis. Using elemenatary Calculus terminogy, we can express this fact via the following double limit. $\lim _{l \rightarrow \omega, m \rightarrow \omega} \mathrm{VT}(l, m)=\mathrm{VT}(l \rightarrow \omega, m \rightarrow \omega)=$ $\operatorname{VT}(\omega, \omega)=\mathrm{VT}$. This notation admittedly may be misleading, since it can 
be interpretated as that the limit of a constant sequence whose every term is False is True. This course is blatantly absurd. What is meant by this double limit is rather the following: For $l<l^{\prime} \leq \omega$ and $m \leq m^{\prime}$ with $m<l$ and $m^{\prime}<l^{\prime}, \mathrm{VT}(l, m)$ and $\mathrm{VT}\left(l^{\prime}, m^{\prime}\right)$ are both false, but the last is closer to the truth. At the limit, it becomes actually true. For $2<n \leq l<m<\omega$, $\operatorname{VT}(l, m)$ is not regarded in this context as False nor True, but rather having a 'fuzzy' value if you like, or $\mathrm{VT}(\mathrm{I}, \mathrm{m})$ is a probablity function whose values are between 0 and 1 . The fuzziness decreases and the probability increases to reach certainty, namely, probability 1, asserting that Atomic countable theories have countable models, namely, that VT holds for $L_{\omega, \omega}$. Having said that, perhaps the more suitable notation would be the (double) $\sum_{m} \sum_{l} \mathrm{VT}(l, m)=\mathrm{VT}$.

\section{References}

[1] H. Andréka, M. Ferenczi, I. Németi (eds.), Cylindric-like Algebras and Algebraic Logic, vol. 22 of Bolyai Society Mathematical Studies, Springer Verlag (2013), DOI: https://doi.org/10.1007/978-3-642-35025-2.

[2] H. Andrka, I. Nmeti, T. Sayed Ahmed, Omitting types for finite variable fragments and complete representations of algebras, Journal of Symbolic Logic, vol. 73(1) (2008), pp. 65-89, DOI: https://doi.org/10.2178/ jsl/1208358743.

[3] B. Bir, Non-finite-axiomatizability results in algebraic logic, Journal of Symbolic Logic, vol. 57(3) (1992), pp. 832-843, DOI: https://doi.org/ $10.2307 / 2275434$.

[4] P. Blackburn, M. d. Rijke, Y. Venema, Modal Logic, Cambridge Tracts in Theoretical Computer Science, Cambridge University Press (2001), DOI: https://doi.org/10.1017/CBO9781107050884.

[5] J. Bulian, I. Hodkinson, Bare canonicity of representable cylindric and polyadic algebras, Annals of Pure and Applied Logic, vol. 164(9) (2013), pp. 884-906, DOI: https://doi.org/10.1016/j.apal.2013.04.002.

[6] A. Daigneault, J. Monk, Representation Theory for Polyadic algebras, Fundamenta Mathematicae, vol. 52 (1963), pp. 151-176, DOI: https: //doi.org/10.4064/fm-52-2-151-176. 
[7] P. Erds, Graph Theory and Probability, Canadian Journal of Mathematics, vol. 11 (1959), pp. 34-38, DOI: https://doi.org/10.4153/CJM-1959-003-9.

[8] M. Ferenczi, A new representation theory: Representing cylindric-like algebras by relativized set algebras, [in:] H. Andréka, M. Ferenczi, I. Németi (eds.), Cylindric-like Algebras and Algebraic Logic, vol. 22 of Bolyai Society Mathematical Studies, Springer Verlag (2013), pp. 135-162, DOI: https://doi.org/10.1007/978-3-642-35025-2_7.

[9] D. H. Fremlin, Consequences of Martin's Axiom, Cambridge Tracts in Mathematics, Cambridge University Press (1984), DOI: https://doi.org/10. 1017/CBO9780511896972.

[10] L. Henkin, J. D. Monk, A. Tarski, L. Henkin, J. D. Monk, A. Tarski, Cylindric Algebras. Part I, Journal of Symbolic Logic, vol. 50(1) (1985), pp. 234-237, DOI: https://doi.org/10.2307/2273803.

[11] R. Hirsch, I. Hodkinson, Complete representations in algebraic logic, Journal of Symbolic Logic, vol. 62(3) (1997), pp. 816-847, DOI: https://doi.org/10.2307/2275574.

[12] R. Hirsch, I. Hodkinson (eds.), Relation algebras by games, vol. 147 of Studies in Logic and the Foundations of Mathematics, Elsevier Science, Amsterdam (2002).

[13] R. Hirsch, I. Hodkinson, Completions and complete representations, [in:] H. Andréka, M. Ferenczi, I. Németi (eds.), Cylindric-like Algebras and Algebraic Logic, vol. 22 of Bolyai Society Mathematical Studies, Springer Verlag (2013), pp. 61-90, DOI: https://doi.org/10.1007/978-3-642-35025-2 4.

[14] R. Hirsch, I. Hodkinson, A. Kurucz, On modal logics between $K \times K \times$ $K$ and $S 5 \times S 5 \times S 5$, Journal of Symbolic Logic, vol. 67(1) (2002), pp. 221-234, DOI: https://doi.org/10.2178/jsl/1190150040.

[15] I. Hodkinson, Atom structures of cylindric algebras and relation algebras, Annals of Pure and Applied Logic, vol. 89(2) (1997), pp. 117-148, DOI: https://doi.org/10.1016/S0168-0072(97)00015-8.

[16] A. Kurucz, Representable cylindric algebras and many dimensional modal logics, [in:] H. Andréka, M. Ferenczi, I. Németi (eds.), Cylindric-like Algebras and Algebraic Logic, vol. 22 of Bolyai Society Mathematical Studies, Springer Verlag (2013), pp. 185-204, DOI: https://doi.org/10.1007/9783-642-35025-2_9. 
[17] R. D. Maddux, Nonfinite axiomatizability results for cylindric and relation algebras, Journal of Symbolic Logic, vol. 54(3) (1989), pp. 951-974, DOI: https://doi.org/10.2307/2274756.

[18] T. Sayed Ahmed, Amalgamation for reducts of polyadic algebras, Algebra Universalis, vol. 51 (2004), pp. 301-359, DOI: https://doi.org/10.1007/ s00012-004-1807-y.

[19] T. Sayed Ahmed, Completions, Complete representations and Omitting types, [in:] H. Andréka, M. Ferenczi, I. Németi (eds.), Cylindric-like Algebras and Algebraic Logic, vol. 22 of Bolyai Society Mathematical Studies, Springer Verlag (2013), pp. 205-221, DOI: https://doi.org/10.1007/978-3642-35025-2_10.

[20] T. Sayed Ahmed, Neat reducts and neat embeddings in cylindric algebras, [in:] H. Andréka, M. Ferenczi, I. Németi (eds.), Cylindric-like Algebras and Algebraic Logic, vol. 22 of Bolyai Society Mathematical Studies, Springer Verlag (2013), pp. 105-134, DOI: https://doi.org/10.1007/978-3642-35025-2_6.

[21] T. Sayed Ahmed, The class of completely representable polyadic algebras of infinite dimensions is elementary, Algebra Universalis, vol. 72(1) (2014), pp. 371-390, DOI: https://doi.org/10.1007/s00012-014-0307-y.

[22] T. Sayed Ahmed, On notions of representability for cylindric-polyadic algebras, and a solution to the finitizability problem for quantifier logics with equality, Mathematical Logic Quarterly, vol. 61(6) (2015), pp. 418-477, DOI: https://doi.org/10.1002/malq.201300064.

[23] T. Sayed Ahmed, Representability for cylindric and polyadic algebras, Studia Mathematicea Hungarica, vol. 56(3) (2019), pp. 335-363.

[24] S. Shelah, Classification Theory, vol. 92 of Studies in Logic and the Foundations of Mathematics, Elsevier (1978).

[25] Y. Venema, Atom structures and Sahlqvist equations, Algebra Universalis, vol. 38 (1997), pp. 185-199, DOI: https://doi.org/10.1007/s000120050047.

\section{Tarek Sayed Ahmed}

Cairo University

Department of Mathematics

Faculty of Science

Giza, Egypt

e-mail: rutahmed@gmail.com 